\title{
Modeling Thermal Systems With Fractional Models: Human Bronchus Application
}

\section{Jean-François Duhé}

University of Bordeaux: Universite de Bordeaux

stephane victor ( $\nabla$ stephane.victor@ims-bordeaux.fr )

UMR5218: Laboratoire de l'integration du materiau au systeme https://orcid.org/0000-0002-0575-0383

\section{Pierre Melchior}

Bordeaux INP

\section{Youssef Abdelmoumen}

IHU Liryc

François Roubertie

INSERM U1045

\section{Research Article}

Keywords: Heat equation, two-port network, thermal impedance, thermal systems, biological systems, constant phase element (CPE), fractional calculus, fractional systems, lung thermal modeling

Posted Date: June 1st, 2021

DOl: https://doi.org/10.21203/rs.3.rs-430449/v1

License: (c) (i) This work is licensed under a Creative Commons Attribution 4.0 International License. Read Full License

Version of Record: A version of this preprint was published at Nonlinear Dynamics on January 28th, 2022. See the published version at https://doi.org/10.1007/s11071-022-07239-3. 


\title{
Modeling thermal systems with fractional models: human bronchus application
}

\author{
Jean-François Duhé · Stéphane Victor • Pierre Melchior • Youssef \\ Abdelmounen • François Roubertie
}

Received: date / Accepted: date

\begin{abstract}
Thermal modeling of systems allows heat and temperature simulations for many applications, such as refrigeration design, heat dissipation in power electronics, melting processes and bio-heat transfers. Sufficiently accurate models are especially needed in openheart surgery where lung thermal modeling will prevent pulmonary cell dying. For simplicity purposes, simple $\mathrm{RC}$ circuits are often used but such models are too simple and lack of precision in dynamical terms. A more complete description of conductive heat transfer can be obtained from the heat equation by means of a twoport network. The analytical expressions obtained from such circuit models are complex and nonlinear in the frequency $\omega$. This complexity in Laplace domain is difficult to handle when it comes to control applications and more specifically during surgery, as heat transfer and temperature control of a tissue may help in reducing necrosis and preserving a greater amount of a given organ. Therefore, a frequency domain analysis of the series and shunt impedances will be presented and different techniques of approximations will be explored in order to obtain simple but sufficiently precise linear fractional transfer function models. Several approximations are proposed to model heat transfers of a human
\end{abstract}

J.-F. Duhé, S. Victor and P. Melchior

at Univ. Bordeaux, CNRS, IMS-UMR 5218,

Bordeaux INP/enseirb-matmeca,

351 cours de la Libération,

33405 Talence Cedex France

Tel.: +33540003627

E-mail: stephane.victor@ims-bordeaux.fr

- J.-F. Duhé, Y. Abdelmounen and F. Roubertie

IHU Liryc, Electrophysiology and Heart Modeling Institute, Fondation Bordeaux Université 33000 Bordeaux Cedex France

INSERM, Centre de recherche Cardio-Thoracique de Bordeaux, 33000 Bordeaux Cedex France middle bronchus and will be quantified by the absolute errors.

Keywords Heat equation · two-port network · thermal impedance · thermal systems · biological systems · constant phase element $(\mathrm{CPE}) \cdot$ fractional calculus · fractional systems · lung thermal modeling

\section{Introduction}

Thermal modeling of systems is of particular interest in applications where temperature might be critical. This includes air conditioning, industrial refrigeration, electronic device cooling, biological tissue heat losses etc. The complexity of heat transfer usually implies the use of finite element methods to solve the heat equation in a chosen region of space. However, finite element models tend to be complex to calculate and in many cases a simpler model that only takes into account temperatures around particular points of interest are precise enough for specific applications.

A typical simple model to take into account heat dissipation and its dynamics is the RC circuit. It is usually used in the domain of power electronics [16], building simulation $[26,8]$ and even to model human heat losses $[14,13]$. RC circuit models are also present in other similar applications, such as the measurement of bio-impedances [5] or lithium-ion battery models [32]. In most of these applications, the system input is assumed a low frequency signal. In order to widen the frequency range and especially in high frequency, the thermal two-port network was introduced in [19] and such a model is directly derived from the heat equation. 
The thermal two-port network models heat conduction in a single direction as a $\mathrm{T}$ circuit (see figure 2) with two series impedances $Z_{1}(s)$ and $Z_{2}(s)$ and a shunt impedance $Z_{3}(s)$. These impedance expressions are complex and nonlinear in $\omega$ which do not allow obtaining rational transfer functions, the latter being more suited models for control design. It can be shown in high frequency that the thermal impedance of a plane wall is given by a half-order integrator $[2,21]$ :

$\lim _{\omega \rightarrow \infty} Z_{\text {thermal }}(j \omega)=\lim _{\omega \rightarrow \infty} \frac{T(j \omega)}{\phi(j \omega)}=\frac{K}{\sqrt{j \omega}}$.

Such an expression shows that the heat transfer in high frequency may be modelled by using constantphase elements (CPE) (see $[15,25,30])$. Constant-phase elements have particularly been used in biological and medical applications such as modeling intestine tissue [12], porous films [9], cardiac tissue [18] or even lung mechanics [6], thus showing the growing importance of fractional calculus in modeling applications.

During surgery, heat transfer and temperature control of a tissue may help in reducing necrosis and preserving a greater amount of a given organ. Patients can suffer from lung injuries during cardiopulmonary bypass, such as ischemia [3]. Perfusion techniques involving some type of indirect temperature control exist [20]. Some lung injuries may be treated by applying mild hypothermia [29]. The precision required to keep a fairly precise temperature in the tissue justifies our interest in enhancing the knowledge regarding the dynamics of thermal models for physiological scenarios.

The contributions of this paper lies on at giving different approximation models for both the series and shunt impedances. The series impedances will be approximated by means of an asymptotic model (taking into account only low and high-frequency behavior), pole-zero cells and the use of a fractional Butterworth impedance model. On the other hand, the shunt impedance will be approximated by a capacitance (its most basic model) and by filtered capacitance models: fractional slope filter and multiple pole filters. The poles for the last filter will be analyzed with and without a recursive factor imposed between the parameters. All these model approximations for the series and shunt impedances will be tested on a biological system: the heat transfer in a human bronchus.

This paper is organized as follows. The presentation of the thermal two-port network and its frequencydomain analysis will be presented in section 2 . The approximation propositions will be explored in section 3 by providing simulation results. A biological application by modeling the human bronchus will be presented in section 4. Finally, section 5 presents conclusions, final remarks and research perspectives.

\section{The analytical thermal two-port network}

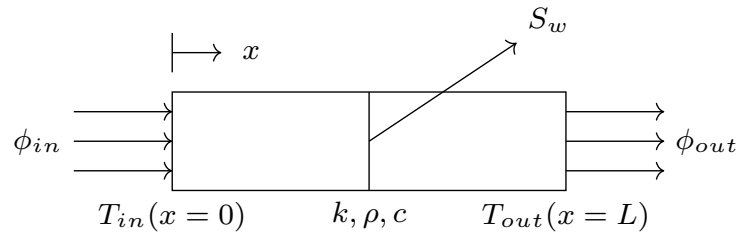

Fig. 1 1D thermal system

Let us consider the heat conduction on a simple plane wall in its longitudinal direction $x$, as shown in figure 1 where $k, \rho$ and $c$ respectively stand for the medium thermal conductivity, density and heat capacity. The total length is $L$ and the cross section is $S_{w}$. The heat equation is expressed as:

$\rho c \frac{\partial T}{\partial t}=k \nabla^{2} T$

and as conduction only goes in $x$ direction, it can be written as:

$\rho c \frac{\partial T}{\partial t}=k \frac{\partial^{2} T}{\partial x^{2}}$

The Laplace transform of equation (3), with null initial conditions, leads to:

$s T(x, s)=\frac{k}{\rho c} \frac{\partial^{2} T(x, s)}{\partial x^{2}}$,

where $s$ denotes the Laplace variable.

By considering a heat flux input at $x=0$ and a heat flux output at $x=L$, it then comes:

$$
\begin{gathered}
\dot{Q}_{i n}(s)=-\left.k S_{w} \frac{\partial T(x, s)}{\partial x}\right|_{x=0} \\
\dot{Q}_{\text {out }}(s)=-\left.k S_{w} \frac{\partial T(x, s)}{\partial x}\right|_{x=L},
\end{gathered}
$$

or even, put under a matrix form:

$\left[\begin{array}{c}T_{\text {in }}(s) \\ \dot{Q}_{\text {in }}(s)\end{array}\right]=M\left[\begin{array}{c}T_{\text {out }}(s) \\ \dot{Q}_{\text {out }}(s)\end{array}\right]$

where

$\boldsymbol{M}=\left[\begin{array}{cc}\cosh (\delta L) & \frac{1}{k S_{w} \delta} \sinh (\delta L) \\ k S_{w} \sinh (\delta L) & \cosh (\delta L)\end{array}\right]$

with $\delta=\sqrt{\frac{s}{a}}$ and $a=\frac{\rho c}{k}$. 


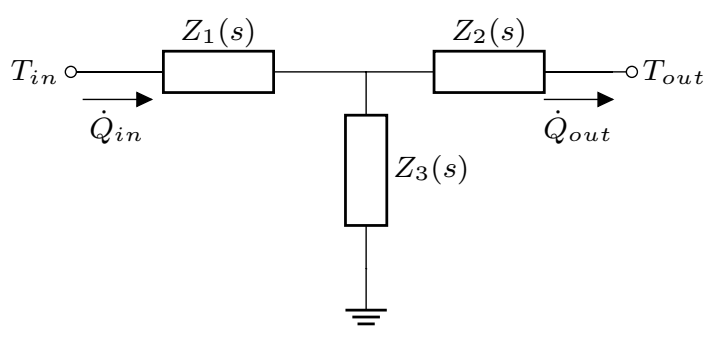

Fig. 2 Thermal two-port network

Note that $\delta$ involves the presence of a fractional (non-integer) operator, as its time-domain representation is a half-order derivative. This matrix equation (6) can be represented by a $\mathrm{T}$ circuit model, such as represented in figure 2.

The series impedances can then be expressed by:

$Z_{1}(s)=Z_{2}(s)=\frac{1}{k S_{w} \delta}[\operatorname{coth}(\delta L)-\operatorname{csch}(\delta L)]$

and the shunt impedance by:

$Z_{3}(s)=\frac{1}{k S_{w} \delta} \operatorname{csch}(\delta L)$

where coth and csch respectively are the hyperbolic cotangent and cosecant functions.

\subsection{Low-frequency behavior}

Processes in thermal applications are usually assumed to be quasi-static, which means that the impedance behavior is only considered in low frequency, thus leading to:

$\lim _{\omega \rightarrow 0} Z_{1}(j \omega)=\frac{L}{2 k S_{w}}=R$

$\lim _{\omega \rightarrow 0} Z_{3}(j \omega)=\frac{1}{\rho c L S_{w} j \omega}=\frac{1}{j \omega C_{t}}$

where $C_{t}=\rho c L S_{w}$.

As it can be seen, low-frequency heat transfers lead to a network consisting only of thermal resistances for the series impedances (which represent energy dissipation) and a capacitance for the shunt impedance (which models energy storage in the medium). This is coherent with the classic RC circuit model used to characterize heat transfer dynamics. However, it should be noted that, depending on the thermal properties of the medium, it is possible to have situations in which the transfer occurs at higher frequencies, which limits the validity of the RC model.

\subsection{High-frequency behavior}

Now, by considering the limits in high frequency, the impedance analysis of the thermal quadrupole, by decoupling them into gain and argument, gives:

$\lim _{\omega \rightarrow \infty}\left|Z_{1}(j \omega)\right|=0, \quad \lim _{\omega \rightarrow \infty} \arg \left|Z_{1}(j \omega)\right|=-45^{\circ}$

and

$\lim _{\omega \rightarrow \infty}\left|Z_{3}(j \omega)\right|=0, \quad \lim _{\omega \rightarrow \infty} \arg \left|Z_{3}(j \omega)\right|=-\infty$.

The impedance $Z_{1}$ clearly exhibits a low-pass filter in high frequency, as shown by its zero gain in high frequency. This suggests a capacitive behavior, but the argument has to be analyzed with more care. As can be observed, the high frequency argument of this impedance is not $-90^{\circ}$, but its half. This allows us to deduce that the high frequency behavior of the series impedance $Z_{1}$ is a constant phase element of phase $-45^{\circ}$ or in other words:

$Z_{1-H F}(s)=\frac{1}{C_{s} s^{0.5}}$.

At first, the shunt impedance $Z_{3}$ seems coherent with the capacitance model in low frequency, as it is normal for a capacitance to exhibit zero gain in high frequency. The argument, on the other hand, suggests that there is an additional filtering that appears in high frequency, which explains why this argument is not the expected $-90^{\circ}$. The high frequency model of $Z_{3}$ can be a combination of a capacitance and a filter, such as:

$Z_{3-H F}(s)=\frac{1}{C_{t} s} H_{\text {filter }}(s)$.

Remark: Adding a correction filter will provide a wider validity domain for the approximation of $Z_{3}$, but it is actually impossible to design a filter that will allow an infinite frequency-range: theoretically, such a filter would have an infinite number of poles in order to obtain the argument shown in equation (13).

\section{Impedance approximations}

It is impossible to well approximate the quadrupole by a model valid for any frequency just by using transfer function models, unless having an infinite number of parameters. However, a truncated transfer function model may be a fairly useful approximation with a wider validity compared to RC circuits. Consequently, a model going from low frequency (or even a static case) to a high but finite upper frequency will be developed. An academic example will be first used to illustrate the main ideas of section 3 and a more complex example 
Table 1 Academic example simulation parameters

\begin{tabular}{cc}
\hline Parameter & Value \\
\hline \hline$a$ & $1 \mathrm{~m}^{2} \cdot \mathrm{s}^{-1}$ \\
\hline$k$ & $1 \mathrm{~W} \cdot \mathrm{m}^{-1} \cdot K^{-1}$ \\
\hline$L$ & $1 \mathrm{~m}$ \\
\hline$S_{w}$ & $1 \mathrm{~m}^{2}$ \\
\hline
\end{tabular}

will be described in section 4 . The academic model parameters are presented in table 8 .

The proposed approximations will require the determination of different parameters in order to be as close as possible to the true impedance behavior. A common quadratic error criterion will be used by taking the error of the gain in $d B$ defined as:

$J(\theta)=\frac{\sum_{i=1}^{N} \lambda^{2(N-i)}\left[\left|Z\left(j \omega_{i}\right)\right|_{d B}-\left|Z_{a p p}\left(j \omega_{i}, \theta\right)\right|_{d B}\right]^{2} \Delta_{i}}{\log \omega_{N}-\log \omega_{1}}$

where $\omega=\left[\omega_{1}, \ldots, \omega_{N}\right]$ is a frequency vector where $\omega_{i} \in[0.1,100 \mathrm{rad} / \mathrm{s}]$ for $i=1, \ldots, N$ and $\lambda$ is a weighting coefficient $(0<\lambda<1)$ that provides a weight for low or high frequencies. If $\lambda=1$, all frequencies have the same weight. Unless indicated, it will be taken as unity. This criterion (16) measures the error in the gain curve given in $d B$.

The series impedance approximations will be optimized by using the Flower Pollination Algorithm (FPA) (see [31] for the complete description), which is a novel meta-heuristic optimization method. The parameters to be tuned in order to apply FPA are pretty low, which makes it faster to tune and test when compared to other algorithms. The main parameters to be tuned are the population size $n$ (normally between 10-25) and a switching probability $p$ which states the possibility of doing one of the two principal operations of the algorithm in an iteration: global pollination or local pollination. The most critical parameter is the switching probability, but applications found in literature tend to use a value of 0.8 [31]. A mathematical study [17] stated that, even though the value of $p$ does not degrade the results when it comes to unimodal functions, a range between 0.5 and 0.8 is preferred. For this study, all optimizations were performed by using $n=10$ and $p=0.8$. The main steps for classic FPA are shown in Algorithm 1.

Remark: The choice of FPA as an optimization tool was mainly due to its simple structure and low parameters to be tuned. However, it should be noted that any other optimization algorithm could also be used to approximate the parameters, such as genetic algorithm or particle swarm optimization.

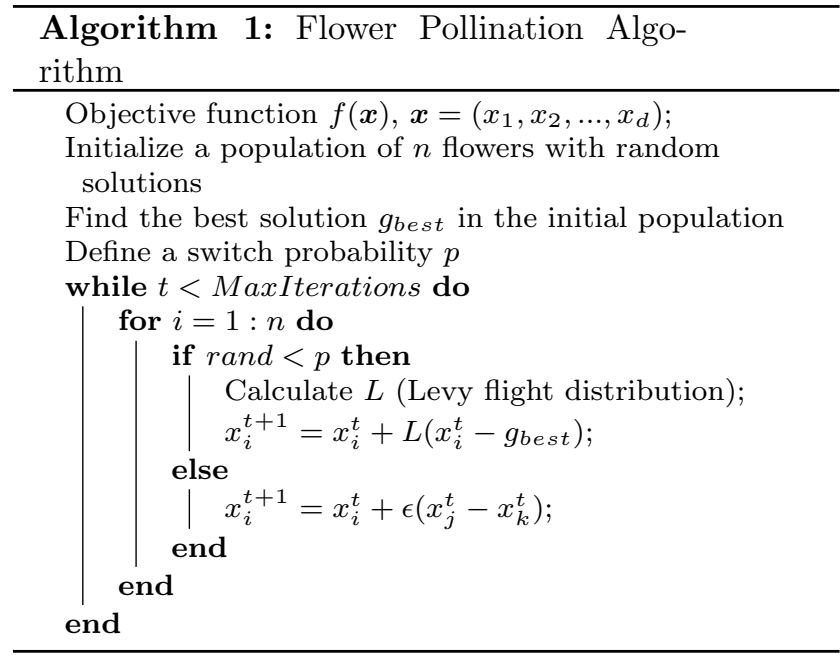

\subsection{Approximation of the series impedances $Z_{1}$ and $Z_{2}$}

\subsubsection{Asymptotic approximation}

As it was seen in the previous section, the series impedance $Z_{1}$ and $Z_{2}$ are equal and behave like a resistance for low frequencies and like a constant phase element at high frequencies. For simplicity sake, only $Z_{1}$ is used. A simple approximation of $Z_{1}$ can be defined as the parallel combination of a resistance and a fractance, such as:

$Z_{1-\text { asymp }}(s)=\frac{R}{1+R C_{s} \sqrt{s}}$,

with $R=\frac{L}{2 k S_{w}}=0.5 \mathrm{~W} \cdot K^{-1}$ and $C_{s}=\frac{k S_{w}}{\sqrt{a}}=1 \mathrm{~J}$. $K^{-1}$.

This first proposition will be called "asymptotic approximation", as it is obtained by only considering both extremes of the frequency domain. The cut-off frequency obtained at $-3 d B$ under the static gain corresponds to (see appendix $\mathrm{C})$ :

$\omega_{b} \approx \frac{0.2679}{\left(R C_{s}\right)^{2}}$

Figures 3 and 4 respectively show the gain and phase for the approximation $Z_{1-\text { asymp }}$ compared with the exact expression of $Z_{1}$ over a frequency range [0.1-100 rad/s]. As expected, both curves get closer towards the limits in low and high frequencies, however, high gain and phase errors occurs in the middle range frequencies. Another important error by using the approximation $Z_{1-\text { asymp }}$, is the cut-off frequency. The true $-3 d B$ frequency under the static gain is almost one decade greater than the one obtained with the asymptotic approximation, which means this approximation leads to a slower dynamic. 


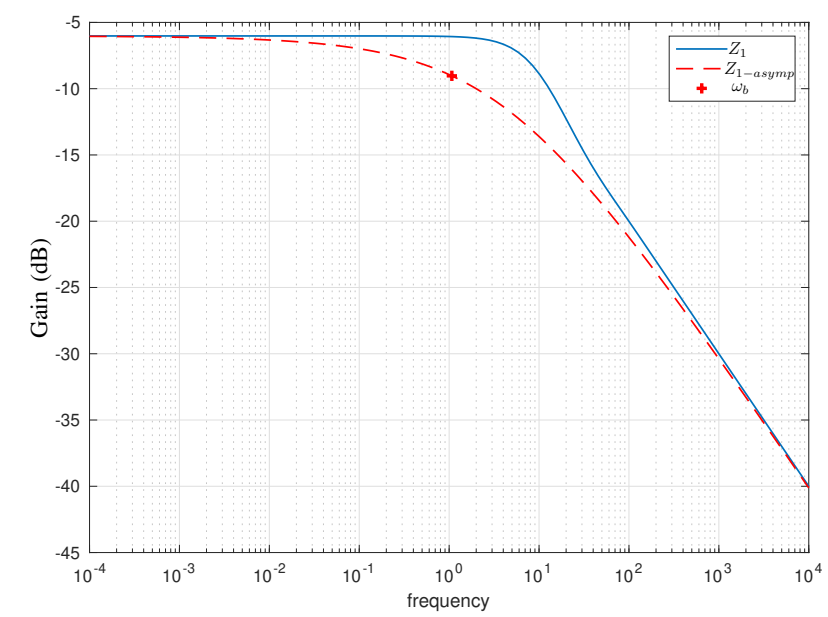

Fig. $3 Z_{1}$ gain diagram and its asymptotic approximation

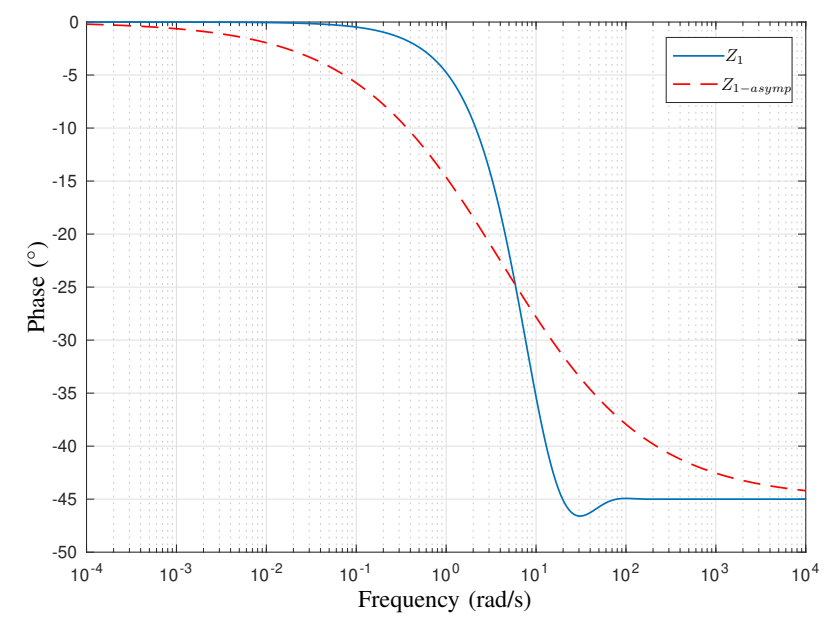

Fig. $4 Z_{1}$ phase diagram and its asymptotic approximation

\subsubsection{Pole-zero approximation}

In order to improve the approximation $Z_{1-\text { asymp }}$, a correction term may be added to the asymptotic approximation. This correction term will adjust the impedance for a mid-band frequency range, as the asymptotic term can properly handle both low and high-frequency limits. This means that the additional term should not contribute to the gain or to the phase in the low or the high-frequency range. A first proposition for the corrected impedance is to use pole-zero cells that will be placed inside the frequency range of interest:

$Z_{1-p z}(s)=\frac{R}{1+R C \sqrt{s}} \prod_{i}^{N_{\text {cells }}} \frac{1+\frac{s}{z_{i}}}{1+\frac{s}{p_{i}}}$.

The parameter vector $\theta$ is given by:

$\theta=\left[\begin{array}{ll}\boldsymbol{p} & \boldsymbol{z}\end{array}\right]$

where $\boldsymbol{p}$ and $\boldsymbol{z}$ are vectors containing all the poles and zeros, respectively.
The number of cells $N_{\text {cells }}$ to be used for the correction will depend on each specific case. Each cell adds a single pole and zero to the transfer function $Z_{1}$. A large number of cells may give pretty accurate approximations, but the thermal model may become too complex and identifiability problems may occur (large number of parameters in the transfer functions).

In order to determine an optimal number of cells to add for the pole-zero approximation, the error criteria $J(\theta)$ was optimized for $N_{\text {cells }}$ going from 1 to 8 in order to estimate an optimal number of poles and zeros to be added. Figure 5 shows the different values for the error criteria.

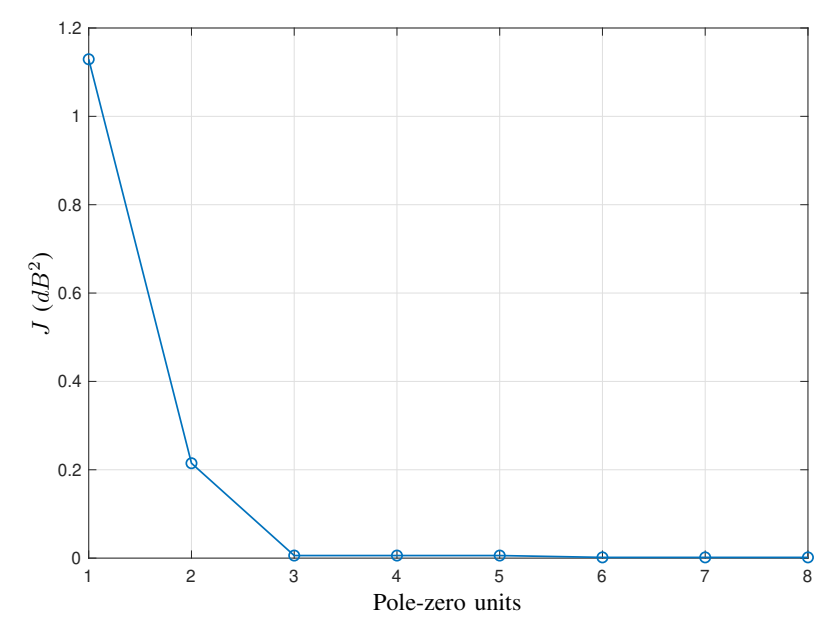

Fig. $5 J$ criterion with varying $N_{\text {cells }}$ for the series impedance correction

As can be seen, no further improvement in the approximation is obtained for this case beyond 3 polezero cells. Even though this technique might succeed in recreating the exact frequency response, the dimension of the parameter vector of the correction term should be taken into account. In this case, $\operatorname{dim}(\theta)=6$. If this thermal impedance is only a section of a global thermal model, it is possible that an approximation of this type will lead to extremely complex expressions for global transfer functions in thermal systems.

\subsubsection{Fractional Butterworth approximation}

By observing the gain plot in figure 3, another approximation can be made for the thermal impedance $Z_{1}$. As it can be seen in the blue curve, the frequency response is considerably flat before the cut-off frequency. This suggests that this impedance behaves like a Butterworth filter [4]. However, the high-frequency gain slope is not an integer multiple of $-20 \mathrm{~dB} / \mathrm{dec}$, but $-10 d B / d e c$. Therefore, inspired by [1], a fractional But- 
terworth filter can be used as an alternative approximation model:

$Z_{1-B W}(s)=\frac{d}{s^{\alpha+\beta}+a s^{\alpha}+b s^{\beta}+c}$.

This new type of model is not a correction term multiplied by the asymptotic approximation, as it can include both the low and high frequency behaviors within its expression:

$\lim _{\omega \rightarrow 0} Z_{1-B W}(j \omega) \approx \frac{d}{c}=R$

and

$\lim _{\omega \rightarrow \infty} Z_{1-B W}(j \omega) \approx \frac{d}{(j \omega)^{\alpha+\beta}}$.

After identification, it is then possible to deduce that:

$\alpha+\beta=0.5$

$c=\frac{1}{R C}$

$d=\frac{1}{C}$

For this type of approximation, the parameter vector will always be limited to 3 parameters:

$\theta=\left[\begin{array}{lll}\alpha & a & b\end{array}\right]$

The fractional Butterworth filter may be a simpler solution with respect to the parameter vector dimension. However, the stability of a system with such a transfer function should be discussed a priori. The BIBO stability for fractional commensurate transfer functions was established by Matignon [22]. An extended criteria for incommensurate systems was later developed [27]. In [1], it is stated that parameters $a$ and $b$ need to be equal or inferior to zero in order to guarantee the fractional stability of the system. The parameters obtained by using the fractional Butterworth filter are given in table 3.1.3.

Table $2 Z_{1}(s)$ approximation parameters for the fractional Butterworth filter

\begin{tabular}{cccc}
\hline \hline$a$ & $b$ & $\alpha$ & $J(\theta)\left(d B^{2}\right)$ \\
\hline 0 & -0.859 & 0.226 & 0.790 \\
\hline
\end{tabular}

It is interesting to observe that parameter $a$ is zero, which simplifies even more the transfer function of the impedance. For this particular case, the thermal impedance obtained is of the form:

$Z(s)=\frac{d}{s^{0.5}+b s^{\beta}+c}$

or even, its thermal admittance is:

$Y(s)=\frac{s^{0.5}}{d}+\frac{b s^{\beta}}{d}+\frac{c}{d}$.

From this last expression, impedance $Z_{1}(s)$ may actually be reinterpreted as being the parallel combination of a half-capacitance, a resistance and fractionalorder new element which can be expressed as:

$Z_{\text {new }}(s)=\frac{G_{0}}{s^{\alpha}} \quad$ with $\quad G_{0}<0$.

Further studies are necessary in order to analyze and give a possible physical meaning to this capacitance-like expression, particularly its negative sign.

\subsubsection{Comparison of the approximations of the series impedance $Z_{1}$}

In order to compare the previous approximations, the absolute error is defined as:

$\epsilon(j \omega)=\left.|| Z_{1-\text { exact }}(j \omega)\right|_{d B}-\left|Z_{1-\text { approx }}(j \omega)\right|_{d B} \mid$.

Figure 6 illustrates the gains for the exact case and the proposed approximations of $Z_{1}$ whereas the error signals are plotted in figure 7 .

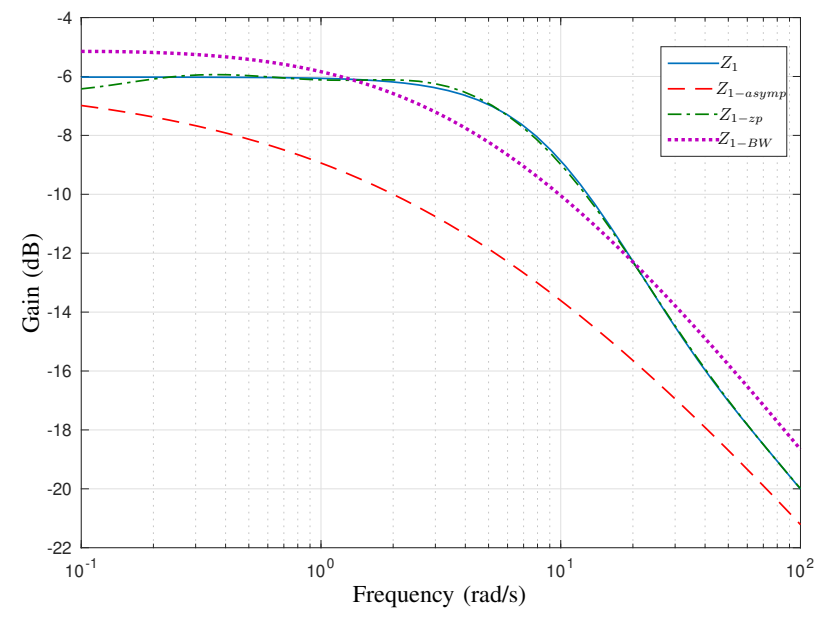

Fig. 6 Gain diagrams of $Z_{1}$ and its approximations

The minimum, maximum and mean values of the error obtained for the three approximations are provided in table 3.1.4. 


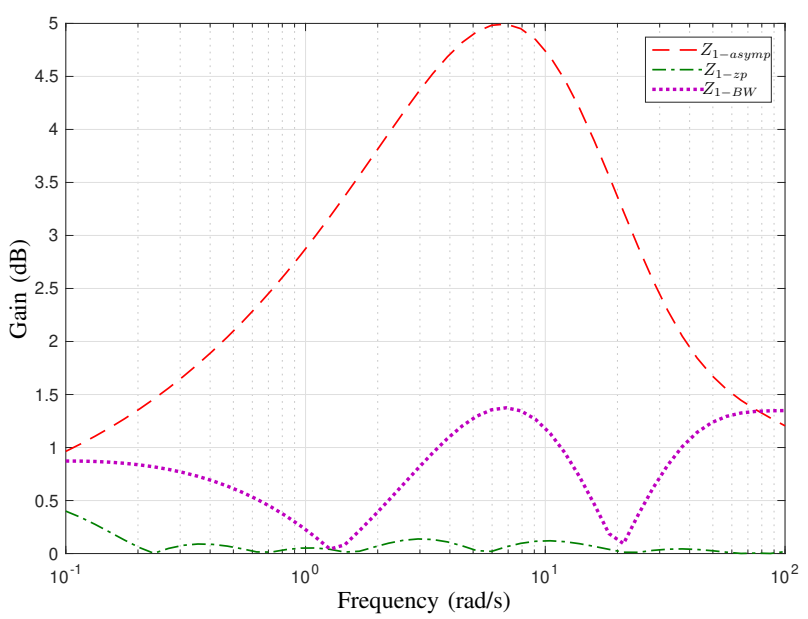

Fig. 7 Absolute errors $\epsilon(j \omega)$ associated with the different approximations of $Z_{1}$

Table 3 Extreme and mean values of the absolute errors obtained for the $Z_{1}$ approximations

\begin{tabular}{cccc}
\hline Approximation & Max $(d B)$ & Mean $(d B)$ & Min $(d B)$ \\
\hline \hline Asymptotic & 4.99 & 2.84 & 0.96 \\
\hline Pole-zero & 0.39 & 0.08 & 0.00 \\
\hline Butterworth & 1.37 & 0.78 & 0.04 \\
\hline
\end{tabular}

Moreover, the mean error for the asymptotic approximation is almost $3 d B$ and the lowest error is around $1 \mathrm{~dB}$, which translates an inaccurate approximation for all mid-range frequencies. When pole-zero cells are added, the mean error is reduced by a factor of 35 , and the error signal can get really close to zero, as shown by its minimum error value (which is not exactly zero, but around $10^{-4}$ ). The Butterworth approximation offers intermediate results, as there is a rough factor of 4 between the asymptotic and the Butterworth mean error. Its peak is still acceptable, but 4 times higher than the one obtained with the polezero approximation. If one needs an extremely precise result, the complexity of the pole-zero cells might be adequate but the simpler Butterworth structure may offer accurate enough results for many applications without adding too much parameters.

\subsection{Approximation of the shunt impedance $Z_{3}$}

\subsubsection{Capacitance approximation}

The shunt impedance $Z_{3}$ behaves like a pure capacitance for low frequencies, but its high frequency behavior is more complex than that of the series impedance, namely:

$Z_{3-c a p}(s)=\frac{1}{C_{t}}$, with $C_{t}=a k L S_{w}=1$.

The gain frequency response of $Z_{3}$ and its capacitance approximation are shown in figure 8 .

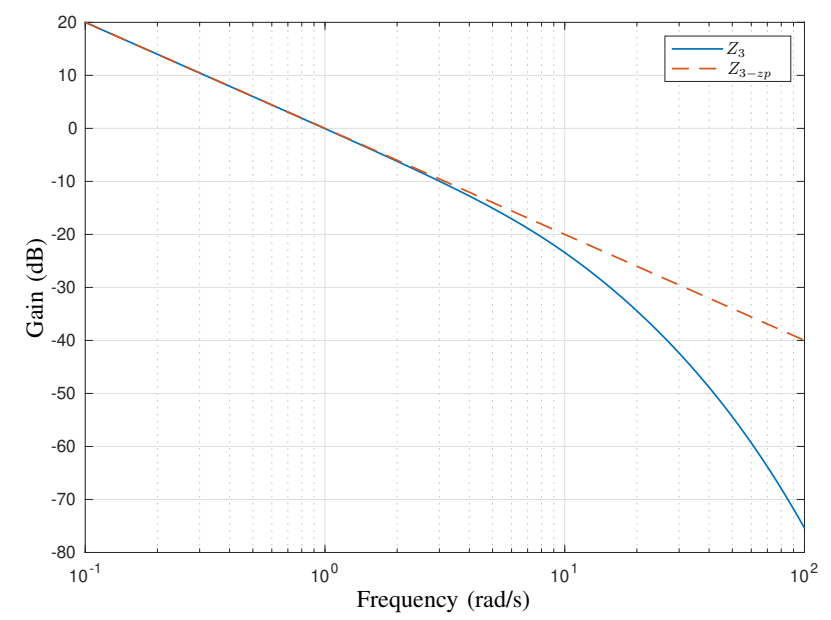

Fig. $8 Z_{3}$ gain diagram and its capacitance approximation

The $-20 d B / d e c$ slope, seen in low frequencies, confirms the capacitance behavior of $Z_{3}$. However, the slope of the gain curve increases as the frequencies increase. Even though a capacitance tends to behave like a shortcircuit on high frequencies, the slope of the gain is not increased and the error curve increases indefinitely in high frequency (see figure 9).

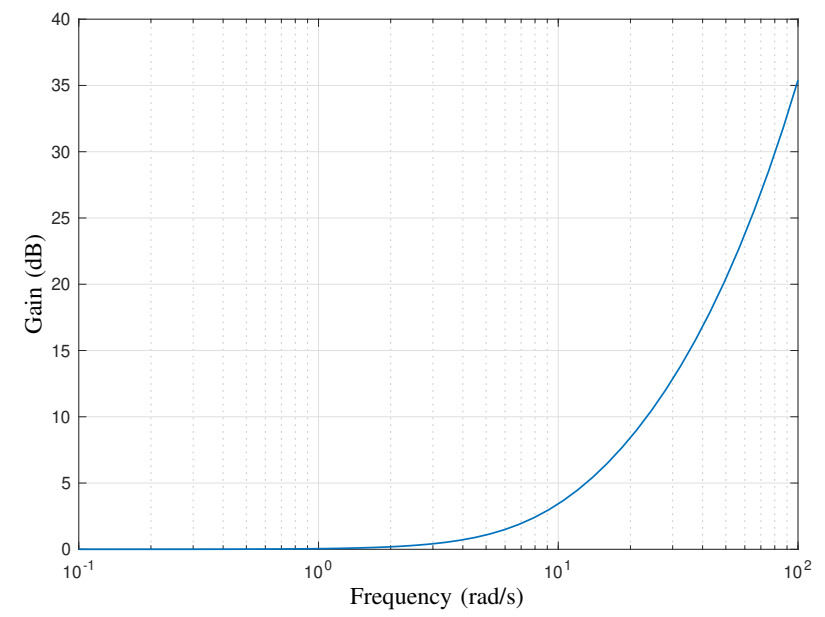

Fig. 9 Absolute error $\epsilon(j \omega)$ associated with the capacitance approximation of $Z_{3}$

\subsubsection{Fractional slope approximation}

If the true thermal system is excited in the mid-range frequencies, it is suggested to modify the classic capacitance model to include a slope increment. 
In this academic example, and by looking at the low and high frequency slopes present in figure 8, one gets:

$\left.\frac{d\left|Z_{3}(j \omega)\right|}{d \omega}\right|_{\omega=0.1}=-20.00 \mathrm{~dB} / \mathrm{dec}$

and

$\left.\frac{d\left|Z_{3}(j \omega)\right|}{d \omega}\right|_{\omega=100}=-78.28 d B / d e c$

The difference between the initial and the final slopes is of almost $-60 \mathrm{~dB} / \mathrm{dec}$. In order to correct this error, a simple fractional filter is proposed as a multiplicative term to the low-frequency capacitance:

$Z_{3-f r a c}(s)=\frac{1}{C_{t} s}\left[\frac{1}{1+(\tau s)^{\phi}}\right]$

This fractional correction term increases the high frequency slope magnitude by the factor $\phi$, namely $20 \phi d B / d e c$ $1 / \tau$ indicates the breaking point from where the added correction term begins to influence. An initial estimate for $\phi$ would be 2.90 in order to be close to the final slope of $-78.28 \mathrm{~dB} / \mathrm{dec}$ around the higher frequency. The parameter vector for this case is:

$\theta_{\text {frac-slope }}=\left[\begin{array}{ll}\tau & \phi\end{array}\right]$

The correction filter term in equation (35) has the following form:

$G(s)=\frac{1}{1+(\tau s)^{\phi}}=\frac{1}{s^{\alpha}+b}$,

a filter that reminds a commensurate transfer function with order $\alpha$ and Matignon's stability theorem may be applied [22], namely:

$\left|\arg \left(\lambda_{i}\right)\right|>\alpha \frac{\pi}{2}$

where, for $\lambda=s^{\alpha}, \lambda_{i}$ are the roots of the characteristic polynomial in $\lambda$.

For the general structure presented in equation (37), we have:

$\lambda_{i}=-b \Rightarrow|\arg (-b)|=\pi$.

Therefore, for this type of filter, the stability condition reads $\alpha<2$ for $b>0$.

\subsubsection{Multiple fractional slope approximation}

According to Matignon stability theorem, the fractional order $\alpha$ being limited to 2 , it may not be possible to go beyond this limit and to get a more suited highfrequency slope. However, a multi fractional-slope filter may also be proposed:

$Z_{3-m u l t-f r a c}(s)=\frac{1}{C_{t} s} \prod_{i=1}^{N} \frac{1}{1+\left(\tau_{i} s\right)^{\nu}}$.

The number of cells to be added $N$ depends on the required additional slope. As each cell may add up to $-40 d B / d e c$, in this case $N=2$.

\subsubsection{Reduced pole approximation}

Looking at the gain curve and its slope in high frequency, an alternative correction term could be the addition of poles to take into account the slope increment.

The difference of slopes in the frequency band of interest, namely:

$\tilde{N}=\left\lfloor\frac{\mid \text { slope }_{\text {init }}-\text { slope }_{\text {final }} \mid}{20}\right\rceil$,

$\lfloor\cdot 7$ denoting the nearest integer function, provides the number of poles to be added. A new approximation of the shunt impedance can be expressed as:

$Z_{3-\text { rec-poles }}(s)=\frac{1}{C_{t} s} \prod_{i=1}^{\tilde{N}} \frac{1}{1+\tau_{i} s}$

with parameter vector:

$\theta_{\text {poles }}=[\boldsymbol{\tau}]$.

If the required number of poles is low enough, one could simply use this approximation and take each parameter $\tau_{i}$ as an additional variable to approximate in order to get the correction term. However, there may be cases in which the number of additional poles might be too high and its determination will be too complex in terms of direct optimization (local minima convergence problem). In this case, an arbitrary relation may be established between poles as a way to reduce parameters:

$\tau_{i+1}=\tau_{1}^{i \gamma}$.

where all the poles are related to the first pole $\tau_{1}$.

The main advantage of this correction is that the parameter vector will always be limited to the following:

$\theta_{\text {red }}=\left[\begin{array}{ll}\tau_{1} & \gamma\end{array}\right]$

The parameter space will always be $2-\mathrm{D}$ in this case. On the other hand, the main drawback is the loss of flexibility with respect to the general pole addition as detailed in paragraph 3.2.3. 


\subsubsection{Comparison of the approximations of the shunt impedance $Z_{3}$}

The approximations obtained by using the fractional slope, the multiple fractional poles and the reduced poles are compared to the pure capacitance model.

Note that to help the convergence of the FPA algorithm for a better fit in high frequency, the weighting factor $\lambda$ was fixed to 0.95 in the criterion (16) for the fractional and multiple fractional slope models. .

Figure 10 illustrates the gains for the exact case and the proposed approximations of $Z_{3}$ whereas the error signals are plotted in figure 11.

The minimum, maximum and mean values of the error obtained for the four approximations are provided in table 3.2.5.

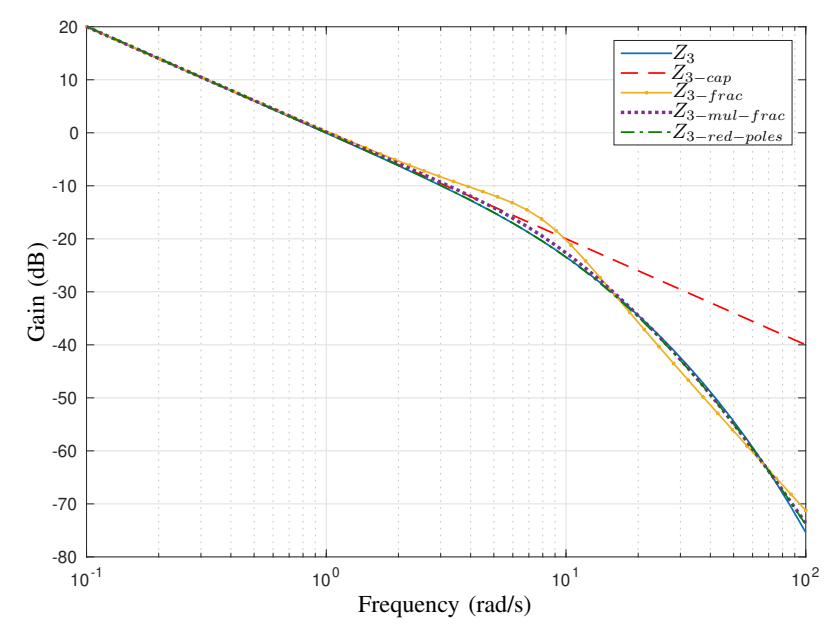

Fig. 10 Gain diagrams of $Z_{3}$ and its approximations

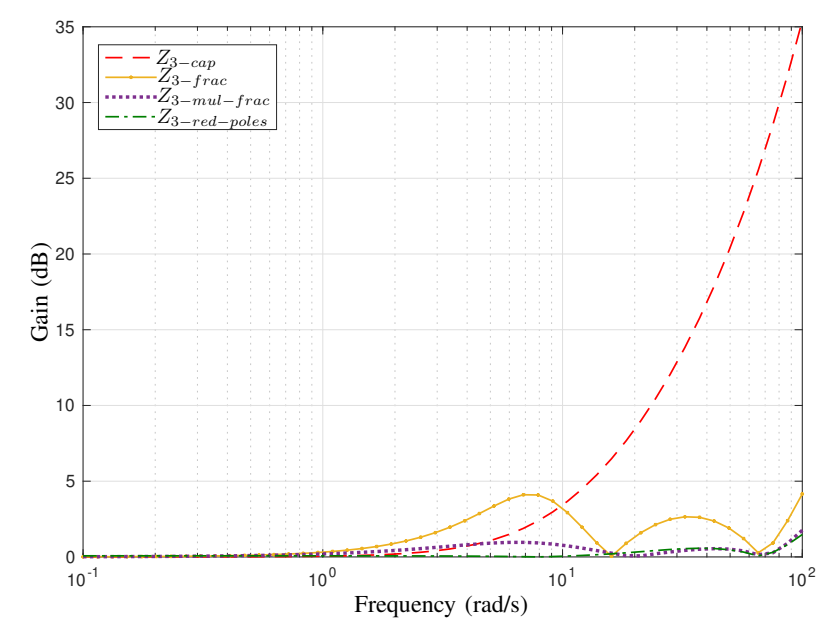

Fig. 11 Absolute errors $\epsilon(j \omega)$ associated with the different approximations of $Z_{3}$
Table 4 Extreme and mean values of the absolute errors obtained for the $Z_{3}$ approximations

\begin{tabular}{cccc}
\hline Approximation & Max $(d B)$ & Mean $(d B)$ & Min $(d B)$ \\
\hline \hline Capacitance & 35.40 & 5.69 & 0.0004 \\
\hline Frac slope & 4.16 & 1.32 & 0.0100 \\
\hline Multi frac slope & 1.76 & 0.39 & 0.0137 \\
\hline Red poles & 1.48 & 0.18 & 0.0027 \\
\hline
\end{tabular}

Even though there is an error that may go up to almost $4.16 d B$ by using the fractional slope, its reduction when compared to the pure capacitance model is evident. For this example, the fractional order was optimized to $\phi=1.45$, which is a rather surprising result. The optimization did not lead to a saturated constraint at $\phi=2$ even though a weight was included for higher frequencies. The obtained value of $1 / \tau=$ $8.22 \mathrm{rad} / \mathrm{s}$ is coherent with the expected the frequency band $[1-10] \mathrm{rad} / \mathrm{s}$ in which the error becomes important (see Figure 11).

Concerning the multiple fractional slope model, the optimization have led to better results. There is roughly a factor of 3 between the mean errors of this model and the simple fractional slope one. The commensurate order was found to be $\nu=1.13$, which means a highfrequency order of $2 \nu=2.26$. The additional flexibility provided by the multiple cell fractional filter permits to go beyond the limit of 2 such as imposed by Matignon's stability criterion in order to get a better fit.

The reduced pole approximation provides even more accurate results and the dimension of the research space for the optimization stays the same. There is a dramatic difference at the point in which the approximation starts to modify the curve, as the recursive pole gave a first pole located at $\tau_{1}=0.033 \mathrm{rad} / \mathrm{s}$, which is two decades below the optimal value obtained by the fractional slope. This approximation may allow a more predictive approach to error reduction than the fractional slope and multiple-cell fractional filter.

\subsubsection{Reduced pole approximation versus all pole approximation}

In view of the previous results, let us consider the latter approximation given in equation (46). Its optimized model can be used as an initial starting point to perform a further optimization: instead of using reduced poles (which indeed has the advantage to reduce the number of parameters in the optimization procedure), this constraint defined in equation (44)) can be released so that all the parameters can be optimized independently. Such an approximation of the shunt impedance 
can be expressed as:

$Z_{3-\text { all-poles }}(s)=\frac{1}{C_{t} s} \prod_{i=1}^{\tilde{N}} \frac{1}{1+\tau_{i} s}$

with the more general parameter vector:

$\theta_{\text {poles }}=[\boldsymbol{\tau}]$,

where all $\tau_{i}$ are independent.

The FPA algorithm was performed with the reduced and all poles by using a gradient descent. As expected, both approximations give really good results. Figure 12 illustrates the comparison of the associated errors for the reduced and the all pole appxoimation cases.

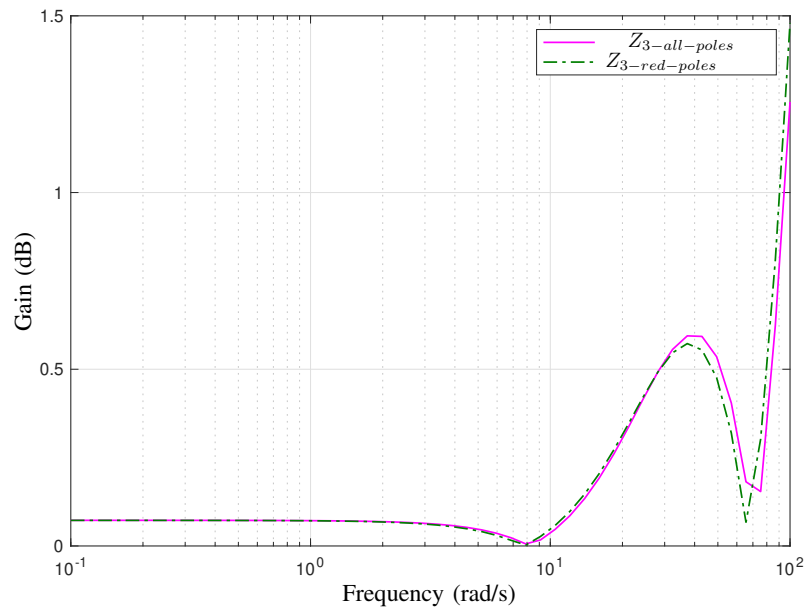

Fig. 12 Absolute errors $\epsilon(j \omega)$ associated with the reduced and all pole approximations of $Z_{3}$

It is indeed difficult to distinguish a further improvement for this new case by allowing the poles to be located at any position. Table 3.2.6 shows the pole locations as well as the criterion $J$ for both cases.

Table 5 Pole location and $J$ criterion for $Z_{3}$ approximations

\begin{tabular}{lcc} 
& Recursive & Non-recursive \\
\hline \hline \multirow{2}{*}{ Poles } & 9.91 & 9.94 \\
& 30.16 & 30.34 \\
$J\left(d B^{2}\right)$ & 98.31 & 92.16 \\
\hline
\end{tabular}

Even if there is a slight improvement regarding the criterion $J$, the non-recursive approximation increased the vector parameter size (three times more than in the recursive case) and the criterion improvement proved to be very slight. On the other hand, it can be seen that all pole approximation locations are almost the same as the ones with the reduced pole approximation. This means that, for this case, the optimal pole location for the approximation was really close to the results provided by the reduced case. It may also justify the use of a reduced pole model in more complex cases.

\section{Application example: heat transfer in a lung bronchus}

A potential application for these approximations is to model heat transfer in the human body. During surgery, heat transfer and temperature control of a tissue may help in reducing necrosis and preserving a greater amount of a given organ.

Patients can suffer from lung injuries during cardiopulmonary bypass, such as ischemia [3]. Perfusion techniques involving some type of indirect temperature control exist [20]. Some lung injuries may be treated by applying mild hypothermia [29]. The precision required to keep a fairly precise temperature in the tissue justifies our interest in enhancing the knowledge regarding dynamic of thermal models for physiological scenarios.

For the application, the heat transfer is considered in the right middle bronchus. Under normal conditions, the nasal cavity and larynx play a significant role regarding air heating and humidification (see [11] and [10]) and procedures impairing this conditioning may change thermal conditions inside the lungs. Human bronchi may be exposed to thermal stress in such conditions. For this analysis, an intermediate length of $L=$ $0.0236 \mathrm{~m}$ was chosen as it is a mean value for right middle bronchus length [24] and radius $r \approx 1 \mathrm{~mm}$ (see [23]).

In terms of frequency, even though human respiration is not a naturally fast mechanism, it may have nonnegligible dynamics for heat transfers. Human breathing can go up to 20 breaths per minute in normal conditions, this breathing being around 12 breaths per minute for an adult in average. Slow breathing is considered in the range $0.07-0.16 \mathrm{~Hz}$ (see [28]) and above 14 breaths per minute (or $0.23 \mathrm{~Hz}$ ), such breathing can already be considered for some as an abnormal value (see [7]). Therefore, the optimization will be carried out in the frequency range $[0.01-5] \mathrm{rad} / \mathrm{s}$ in order to go slightly over a normal condition breathing.

For the series impedance $Z_{1}$, the optimal number of cells for the pole-zero correction was found to be 2 , as shown in figure 13. The gain diagrams of the different approximations proposed in paragraph 3.1 (asymptotic, pole-zero, and fractional Butterworth approximations) and their associated errors are respectively shown in figures 14 and 15. Table 4 summarizes a comparison between the obtained errors.

As expected, the asymptotic approximation offers a significant error for the whole frequency range, which 


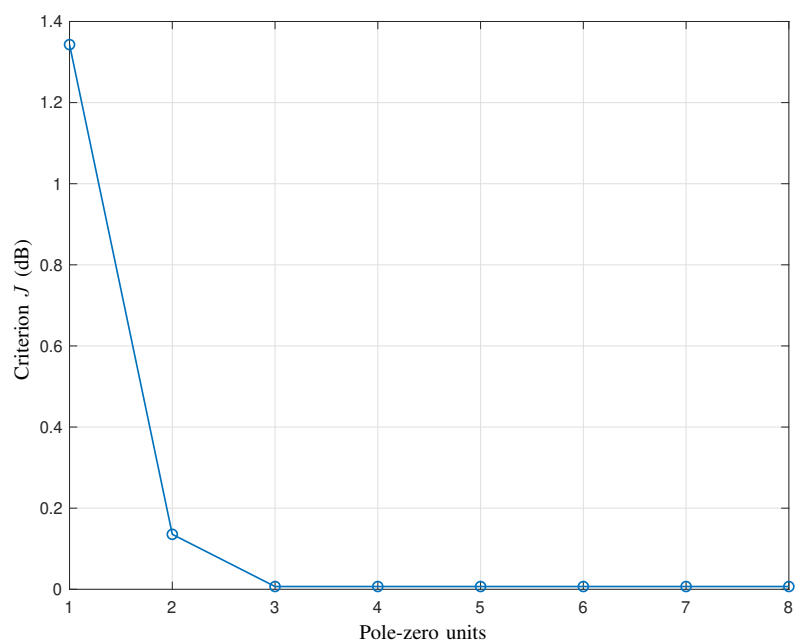

Fig. $13 J$ criterion versus pole-zero units for a human bronchus

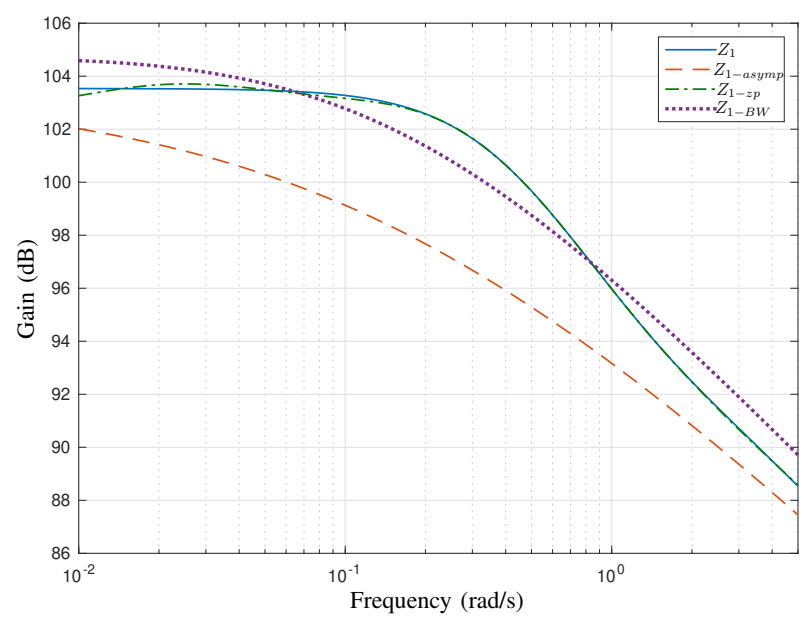

Fig. $14 Z_{1}$ approximation gains for a human bronchus

Table 6 Extreme and mean values of the absolute errors obtained for the $Z_{1}$ approximations of a human bronchus

\begin{tabular}{cccc}
\hline Approximation & $\operatorname{Max}(d B)$ & Mean $(d B)$ & Min $(d B)$ \\
\hline \hline Asymptotic & 4.99 & 3.05 & 1.0829 \\
\hline Pole-zero & 0.26 & 0.05 & 0.0001 \\
\hline Butterworth & 1.34 & 0.80 & 0.0151
\end{tabular}

may indicate that a human bronchus cannot be considered in the low-frequency limit as human breathing cycles are in the mi-band frequency range. Once again, it can be seen that the pole-zero approximation offers the most accurate results. However, for this scenario, the improvement obtained by using the Butterworth is more significant than in the academic case. For this case, the pole-zero approximation has 6 parameters, which includes 3 additional parameters with respect to the Butterworth case. However, the Butterworth impedance still provides an improved estimation than the asymptotic one. It can be seen in Appendix

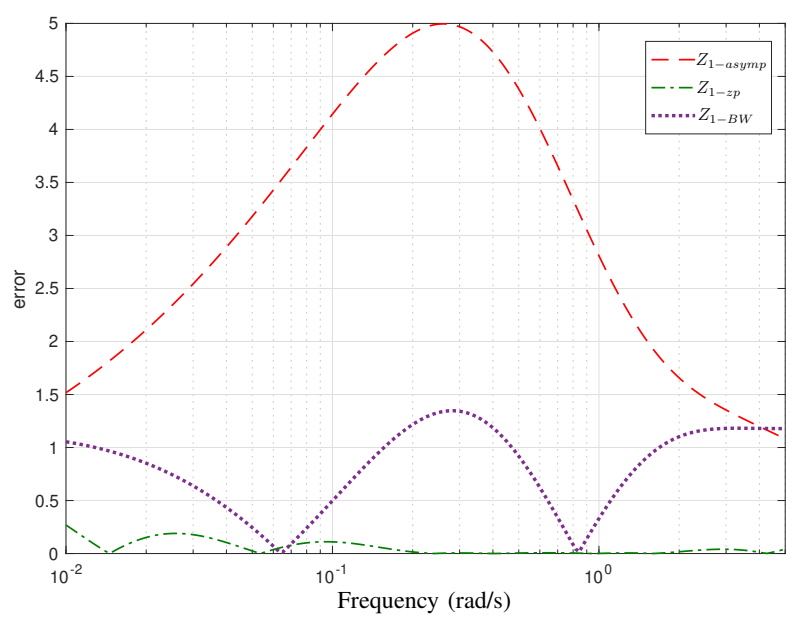

Fig. 15 Absolute errors $\epsilon(j \omega)$ associated with the different approximations of $Z_{1}$ for a human bronchus

$B$ that for this case, the Butterworth impedance was once again reduced to the form shown in equation (28), which offers further advantages in terms of parameter space (2D).

The gain diagrams of the different approximations proposed in paragraph 3.2 (capacitance, fractional slope, multiple fractional slope and pole approximations) and their associated errors are respectively shown in figures 16 and 17. Table 4 summarizes a comparison between the obtained errors.

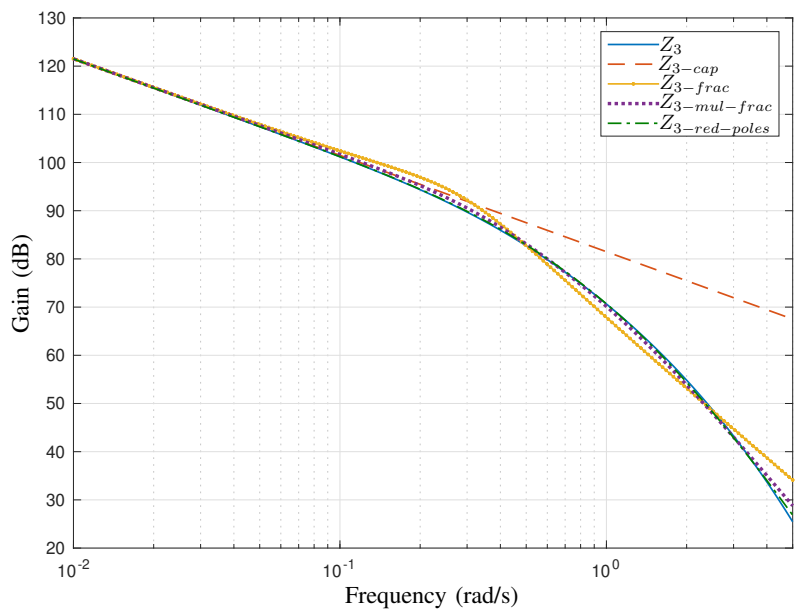

Fig. 16 Gain diagrams of $Z_{3}$ and its approximations for a human bronchus

The pure capacitance model provides the worst approximation: the mean error is of $7.58 \mathrm{~dB}$ in the considered frequency range and it can go up to $42.05 \mathrm{~dB}$ as we get closer to the higher frequency. For this particular geometry, three integer poles were required to provide a good approximation. It should be noted that for a frequency of $1 \mathrm{rad} / \mathrm{s}$ (close to normal breathing), the 


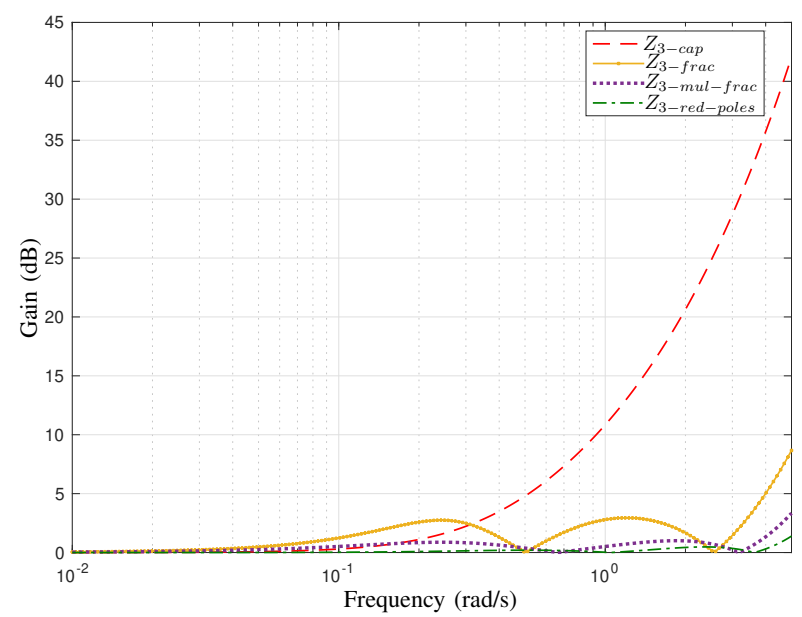

Fig. 17 Absolute errors $\epsilon(j \omega)$ associated with the different approximations of $Z_{3}$ for a human bronchus

Table 7 Error signal peak and mean values for $Z_{3}(s)$ approximations in a human bronchus

\begin{tabular}{lccc}
\hline Approximation & Peak $(d B)$ & Mean $(d B)$ & Min $(d B)$ \\
\hline \hline Capacitance & 42.05 & 7.58 & 0.0031 \\
\hline Frac slope & 8.66 & 1.57 & 0.0494 \\
\hline Multi frac slope & 3.43 & 0.54 & 0.0177 \\
\hline Red poles & 1.39 & 0.14 & 0.0000
\end{tabular}

slope of the $Z_{3}$ gain curve is already $-38 \mathrm{~dB} / \mathrm{dec}$, which means that a normal breathing is already in its midband frequency range. The best results are obtained with the reduced poles approximation with a mean error of $0.14 d B$.

\section{Conclusion and final remarks}

Different propositions for approximating thermal impedances were proposed and compared in terms of accuracy in the frequency ban of interest. The series impedance of a plane wall proved to have an interesting behavior in the frequency domain, as its usual simplification as a simple resistance is inaccurate beyond the lowfrequency spectrum. The presence of a half-capacitance indicates a possible additional thermal accumulation that cannot be taken into account by a simple capacitance model. Even if the resistance-half capacitance parallel model succeeds in approximating the frequency limits, there is a significative error in the mid-band frequency that can be reduced by using pole-zero cells or a fractional Butterworth expression. The pole-zero approximation proved to be better in terms of accuracy, but the impedance expression is complex and the number of parameters required for an optimal approximation may be too large. The pole-zero cells are an exclusively mathematical construct and lack physical mean- ing. The fractional Butterworth approximation may not offer such accurate results, but it still greatly improves the error when compared to the resistance-half capacitance parallel model. The parameter vector is limited to a maximum dimension of 3 and it is suggested that this type of expression may actually be reinterpreted in a circuit model as the parallel addition of additional elements to the resistance-half capacitance model. Additional physical meaning may be obtained from this model in future studies.

The shunt impedance has a more complex frequency behavior and only a low-frequency analysis was done. In low-frequency, this impedance behaves like a capacitance. However, the quick slope decreases (both in gain and phase) in high frequency, a circuit element nor a simple fractional model is sufficient. This is the reason why the approximations presented for $Z_{3}$ are limited to a specific frequency range. The fractional slope helped in reducing the errors and has little parameters to be determined. However, the approximation with recursive poles proved to be more accurate without adding more parameters to the approximation. For the example analyzed, the accuracy was not significantly improved by allowing the poles to be independent. A simple recursive pole approximation gives accurate enough results and provides a simple expression. However, it should be noted that $Z_{1}$ and $Z_{3}$ are inseparable elements of a same system. Therefore, equivalent transfer functions obtained from the $\mathrm{T}$ circuit will involve fractional-order derivatives as it cannot be avoided for the series impedances $Z_{1}$ and $Z_{2}$.

All the proposed approximations for modeling the series and shunt impedance where applied in a simulation example of a human bronchus. As previously stated, the simulation results well illustrates that the pole-zero approximation well fits the series impedance $Z_{1}$ and $Z_{2}$. For the shunt impedance $Z_{3}$, it could be fitted by using a single integer-order pole.

Research perspectives of this study include analyzing different type of scenarios with the thermal twoport networks and its transfer function properties when applying the proposed approximation techniques. The possibility of getting transfer function models with physically meaningful parameters may improve the analysis of system identification for thermal systems.

\section{Declarations}

Funding: Not applicable

Conflicts of interest/Competing interests: The authors have no relevant financial or non-financial interests to disclose.

Code availability (software application or custom code): 
Not applicable

Authors' Contributions - in relation with your submission: J.F. Duhé has developed the results with S. Victor. He also made the simulations. S. Victor, P. Melchior, Y. Abdelmoumen and F. Roubertie have contributed to the biological and physiological results.

Ethics approval (include appropriate approvals or waivers): Not applicable

Consent to participate (include appropriate statements):

Not applicable

Consent for publication (include appropriate statements):

Not applicable

\section{A Estimated transfer functions for the academic example}

Table 8 Estimated models for the academic example of section 3

\begin{tabular}{c||c}
\hline$Z_{1-\text { asymp }}$ & $\frac{0.5}{1+0.5 \sqrt{s}}$ \\
\hline$Z_{1-z p}$ & $\frac{0.5}{1+0.5 \sqrt{s}} \frac{1+\frac{s}{0.2647} \frac{s}{1+\frac{s}{0.378}} \frac{s}{3.446}}{1+\frac{s}{5.73}} \frac{1}{1+\frac{s}{11.21}}$ \\
\hline$Z_{1-B W}$ & $\frac{1}{s^{0.5}-0.859 s^{0.274}+2}$ \\
\hline$Z_{3-\text { cap }}$ & $\frac{1}{s} \frac{1}{1+(0.1097 s)^{1.55}}$ \\
\hline$Z_{3-\text { frac }}$ & $\frac{1}{s+(0.1185 s)^{1.15}} \frac{1+(0.0258 s)^{1.15}}{1+\text { mul-frac }}$ \\
\hline$Z_{3-\text { red-poles }}$ & $\frac{1}{s} \prod_{i=1}^{3} \frac{1}{1+\tau_{i} s} \quad$ with $\tau_{1}=0.033$, \\
$\tau_{2}=\tau_{1}^{\gamma}, \tau_{3}=\tau_{1}^{2 \gamma}$ and $\gamma=0.674$
\end{tabular}

\section{B Estimated transfer functions for human bronchus}

\section{Proof of fractional first-order system bandwidth}

The frequency response of the asymptotic approximation for the series impedance:

$$
Z_{\text {asym }}(j \omega)=\frac{R}{1+R C_{s} \sqrt{j \omega}}
$$

Table 9 Estimated models for the bronchus applications of section 4

\begin{tabular}{c||c}
\hline$Z_{1-\text { asymp }}$ & $\frac{150240}{1+2.5158 \sqrt{s}}$ \\
\hline$Z_{1-z p}$ & $\frac{150240}{1+2.5158 \sqrt{s}} \prod_{1}^{3} \frac{1+\frac{s}{z_{i}}}{1+\frac{s}{p_{i}}}$ \\
& $z_{1}=0.010, z_{2}=0.112, z_{3}=0.981$ \\
\hline$Z_{1-B W}$ & $p_{1}=0.014, p_{2}=0.166, p_{3}=0.528$ \\
\hline$Z_{3-\text { cap }}$ & $\frac{59720.2}{s^{0.5}-0.406 s^{0.25}+0.3975}$ \\
\hline$Z_{3-\text { frac }}$ & $\frac{11869}{s}$ \\
\hline$Z_{3-\text { mul-frac }}$ & $\frac{11869}{s} \frac{1}{1+(0.734 s)^{1.12}} \frac{1+(2.954 s)^{1.12}}{1+(3.3873 s)^{1.36}}$ \\
\hline$Z_{3-\text { red-poles }}$ & $\frac{11869}{s} \prod_{i=1}^{3} \frac{1}{1+\tau_{i} s}$ with $\tau_{1}=2.407$, \\
& $\tau_{2}=\tau_{1}^{\gamma}, \tau_{3}=\tau_{1}^{2 \gamma}$ and $\gamma=-0.438$ \\
\hline
\end{tabular}

from which one gets the gain:

$\left|Z_{\text {asym }}(j \omega)\right|=\frac{R}{\sqrt{\left(R C_{s}\right)^{2} \omega+R C_{s} \sqrt{2 \omega}+1}}$

it can be seen that the highest gain in $d B$ for this system is: $\left|Z_{\text {asym }}(j \omega)\right|_{d B-\max }=20 \log _{10}(R)$

The $-3 d B$ frequency is given by:

$\left|Z_{\text {asym }}\left(j \omega_{b}\right)\right|_{-3 d B}=\left|Z_{\text {asym }}(j \omega)\right|_{d B-\max }-3 d B$

By replacing equation (49) in (51), one gets the following polynomial:

$\left(R C_{s}\right)^{2} \omega_{b}+\sqrt{2} R C_{s} \sqrt{\omega_{b}}+\left(1-10^{0.3}\right)=0$

By taking the single positive and real-valued solution for $\omega_{b}$ :

$\omega_{b} \approx \frac{0.2679}{\left(R C_{s}\right)^{2}}$

which is the expression for the bandwidth.

\section{References}

1. Ali, A., Radwan, A., Soliman, A.: Fractional order butterworth filter: Active and passive realizations. IEEE Journal on Emerging and Selected Topics in Circuits and Systems 3(3), 346-354 (2013). DOI 10.1109/JETCAS.2013.2266753 
2. Battaglia, J., Cois, O., Puigsegur, L., Oustaloup, A.: Solving an inverse heat conduction problem using a noninteger identified model. International Journal of Heat and Mass Transfer 44(14), $2671-2680$ (2001). DOI 10.1016/S0017-9310(00)00310-0

3. Buggeskov, K., Maltesen, R., Rasmussen, B., Hanifa, M., Lund, M., Wimmer, R., Ravn, H.: Lung protection strategies during cardiopulmonary bypass affect the composition of blood electrolytes and metabolites-a randomized controlled trial. Journal of clinical medicine $\mathbf{7}(11), 462$ (2018). DOI $10.3390 / \mathrm{jcm} 7110462$

4. Butterworth, S.: Theory of filter amplifier. Experimental Wireless and the Wireless Engineer 7, 536 - 541 (1930)

5. Chinen, K., Kinjo, I., Zamami, A., Irei, K., Nagayama, K.: New equivalent-electrical circuit model and a practical measurement method for human body impedance. Bio-Medical Materials and Engineering 26, S779-S786 (2015). DOI 10.3233/BME-151369

6. Copot, D.: Fractional calculus based methods and models to characterize diffusion in the human body. Ph.D. thesis, Ghent University (2017)

7. Cretikos, M., Bellomo, R., Hillman, K., Chen, J., Finfer, S., Flabouris, A.: Respiratory rate: the neglected vital sign. Medical Journal of Australia 188(11), 657-659 (2008). DOI 10.5694/j.1326-5377.2008.tb01825.x

8. Danza, L., Belussi, L., Meroni, I., Salamone, F., Floreani, F., Piccinini, A., Dabusti, A.: A simplified thermal model to control the energy fluxes and to improve the performance of buildings. Energy Procedia 101, 97 104 (2016). DOI 10.1016/j.egypro.2016.11.013.

9. Das, S., Sivaramakrishna, M., Das, S., Biswas K.and Goswami, B.: Characterization of a fractional order element realized by dipping a capacitive type probe in polarizable medium. In: Symposium on Fractional Signals and Systems. Lisbon, Portugal (2009)

10. Dias, N., J. R. C., B., Martins, R., Carvalho, L.: Larynx and cervical trachea in humidification and heating of inhaled gases. Annals of Otology, Rhinology \& Laryngology 114(5), 411-415 (2005). DOI $10.1177 / 000348940511400514$

11. Elad, D., Wolf, M., Keck, T.: Air-conditioning in the human nasal cavity. Respiratory Physiology \& Neurobiology 163(1), 121 - 127 (2008). DOI 10.1016/j.resp.2008.05.002

12. Elwakil, A.: Fractional-order circuits and systems: An emerging interdisciplinary research area. IEEE Circuits and Systems Magazine 10(4), 40-50 (2010). DOI 10.1109/MCAS.2010.938637

13. Ismail, N., Ghaddar, N., Ghali, K.: Electric circuit analogy of heat losses of clothed walking human body in windy environment. International Journal of Thermal Sciences 127, 105 - 116 (2018). DOI https://doi.org/10.1016/j.ijthermalsci.2018.01.025

14. Jiang, G., Qu, T., Shang, Z., Zhang, X.: A circuit simulating method for heat transfer mechanism in human body. In: The 26th Annual International Conference of the IEEE Engineering in Medicine and Biology Society, vol. 2, pp. 5274-5276 (2004). DOI 10.1109/IEMBS.2004.1404472

15. Krishna, B.: Studies on fractional order differentiators and integrators: A survey. Signal Processing 91(3), 386 - 426 (2011). DOI 10.1016/j.sigpro.2010.06.022

16. Künzi, R.: Thermal design of power electronic circuits. arXiv: Accelerator Physics 3, 311-327 (2015). DOI 10.5170/CERN-2015-003.311

17. Lukasik, S., Kowalski, P.: Study of flower pollination algorithm for continuous optimization. In: P. Angelov,
K. Atanassov, L. Doukovska, M. Hadjiski, V. Jotsov, J. Kacprzyk, N. Kasabov, S. Sotirov, E. Szmidt, S. Zadrożny (eds.) Intelligent Systems'2014, pp. 451-459. Springer International Publishing, Cham (2015)

18. Magin, R., Ovadia, M.: Modeling the cardiac tissue electrode interface using fractional calculus. 2nd IFAC Workshop on Fractional Differentiation and its Applications 39(11), 302 - 307 (2006). DOI 10.3182/20060719-3-PT4902.00056

19. Maillet, D., André, S., Batsale, J., Degiovanni, A., Moyne, C.: Thermal Quadrupoles: Solving the Heat Equation through Integral Transforms. Loyola Symposium Series. John Wiley \& Sons (2000)

20. Maltesen, R., Buggeskov, K., Andersen, C., Plovsing, R., Wimmer, R., Ravn, H., Rasmussen, B.: Lung protection strategies during cardiopulmonary bypass affect the composition of bronchoalveolar fluid and lung tissue in cardiac surgery patients. Metabolites 8(4), 54 (2018). DOI 10.3390/metabo8040054

21. Malti, R., Sabatier, J., Akçay, H.: Thermal modeling and identification of an aluminium rod using fractional calculus. In: 15th IFAC Symposium on System Identification (SYSID'2009), pp. 958-963. St Malo, France (2009). DOI 10.3182/20090706-3-FR-2004.00159

22. Matignon, D.: Stability properties for generalized fractional differential systems. ESAIM proceedings - Systèmes Différentiels Fractionnaires - Modèles, Méthodes et Applications 5 (1998)

23. McFawn, P., Mitchell, H.: Bronchial compliance and wall structure during development of the immature human and pig lung. European Respiratory Journal 10(1), 2734 (1997)

24. Mi, W., Zhang, C., Wang, H., Cao, J., Li, C., Yang, L., Guo, F., Wang, X., Yang, T.: Measurement and analysis of the tracheobronchial tree in chinese population using computed tomography. PLOS ONE 10(4), 1-14 (2015). DOI 10.1371/journal.pone.0123177

25. Nakagawa, M., Sorimachi, K.: Basic characteristics of a fractance device. IEICE Transactions on Fundamentals of Electronics, Communications and Computer Sciences 75, 1814-1819 (1992)

26. Parnis, G.: Building thermal modelling using electric circuit simulation (2012)

27. Rivero, M., Rogosin, S., Tenreiro Machado, J., Trujillo, J.: Stability of fractional order systems. Mathematical Problems in Engineering 2013, 356215 (2013). DOI $10.1155 / 2013 / 356215$

28. Russo, M.A., Santarelli, D.M., O'Rourke, D.: The physiological effects of slow breathing in the healthy human. Breathe (Sheffield, England) 13(4), 298-309 (2017). DOI 10.1183/20734735.009817

29. Wu, C., Xu, J., Jin, X., Chen, Q., Li, Z., Zhang, M.: Effect of mild hypothermia on lung injury after cardiac arrest in swine based on lung ultrasound. BMC Pulmonary Medicine 19(1), 198 (2019). DOI 10.1186/s12890-0190958-8

30. Yakuphanoglu, F., Krishna, B., Reddy, K.: Active and passive realization of fractance device of order $1 / 2$. Active and Passive Electronic Components 2008, 369421 (2008). DOI 10.1155/2008/369421

31. Yang, X.: Flower pollination algorithm for global optimization. In: J. Durand-Lose, N. Jonoska (eds.) Unconventional Computation and Natural Computation, pp. 240-249. Springer Berlin Heidelberg, Berlin, Heidelberg (2012) 
32. Zhang, L., Peng, H., Ning, Z., Mu, Z., Sun, C.: Comparative research on $\mathrm{RC}$ equivalent circuit models for lithiumion batteries of electric vehicles. Applied Sciences 7(10) (2017). DOI 10.3390/app7101002 
Figures

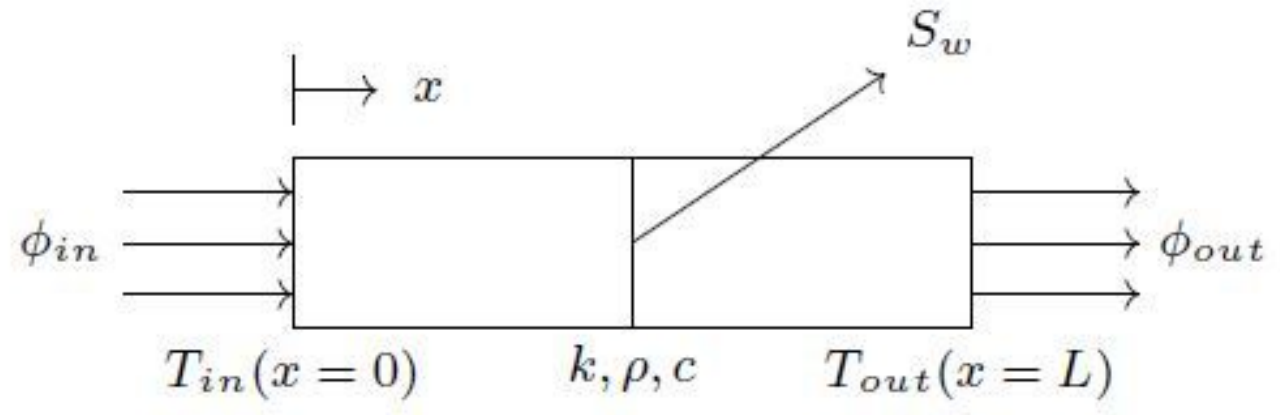

Figure 1

1D thermal system

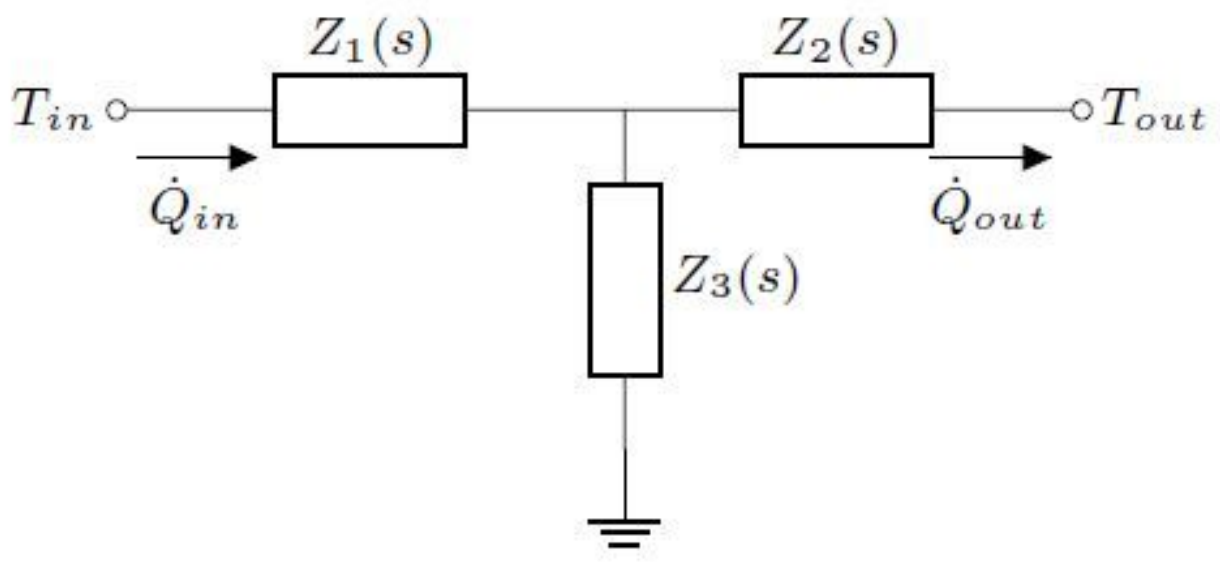

Figure 2

Thermal two-port network 


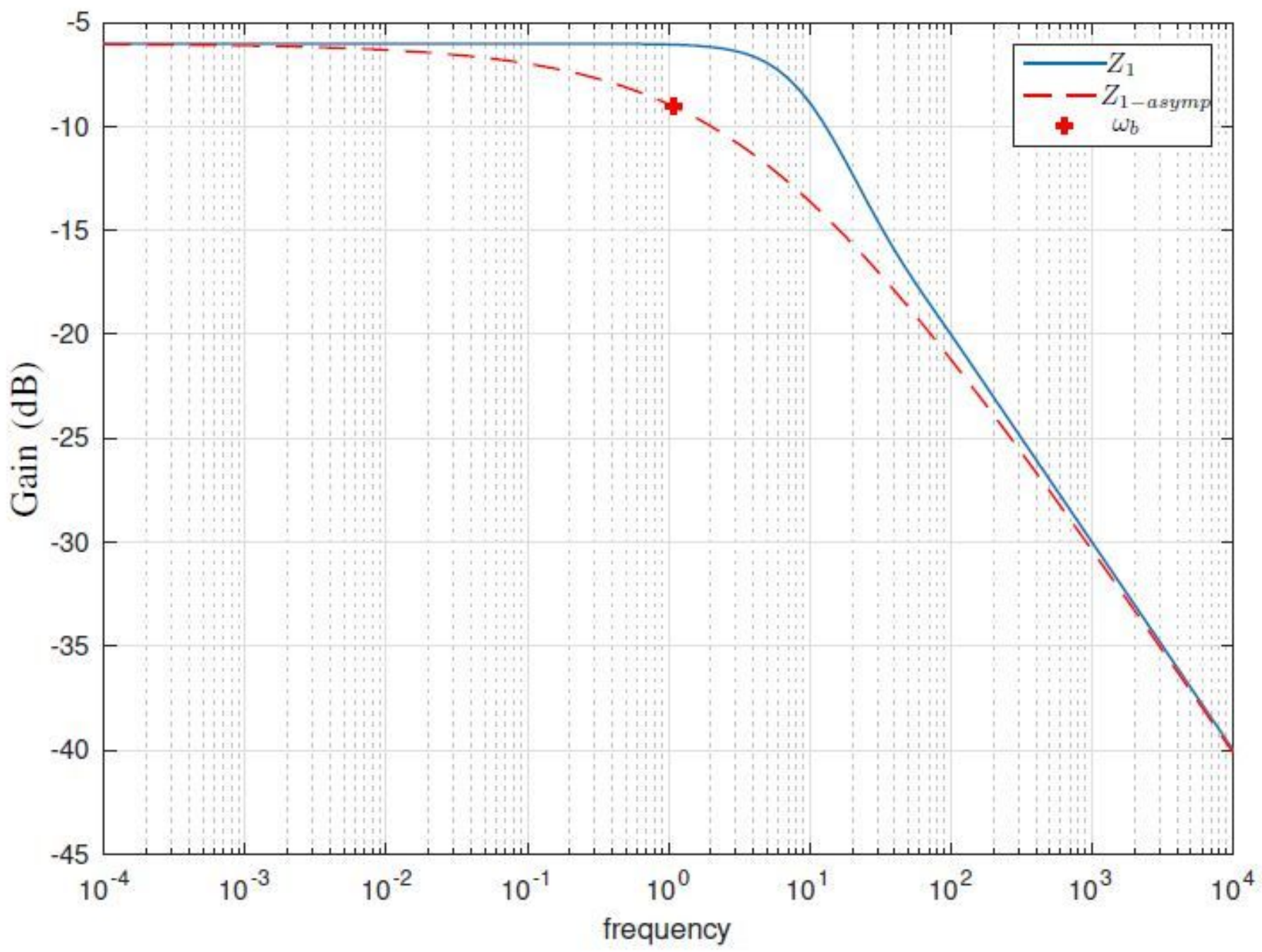

Figure 3

Z1 gain diagram and its asymptotic approximation 


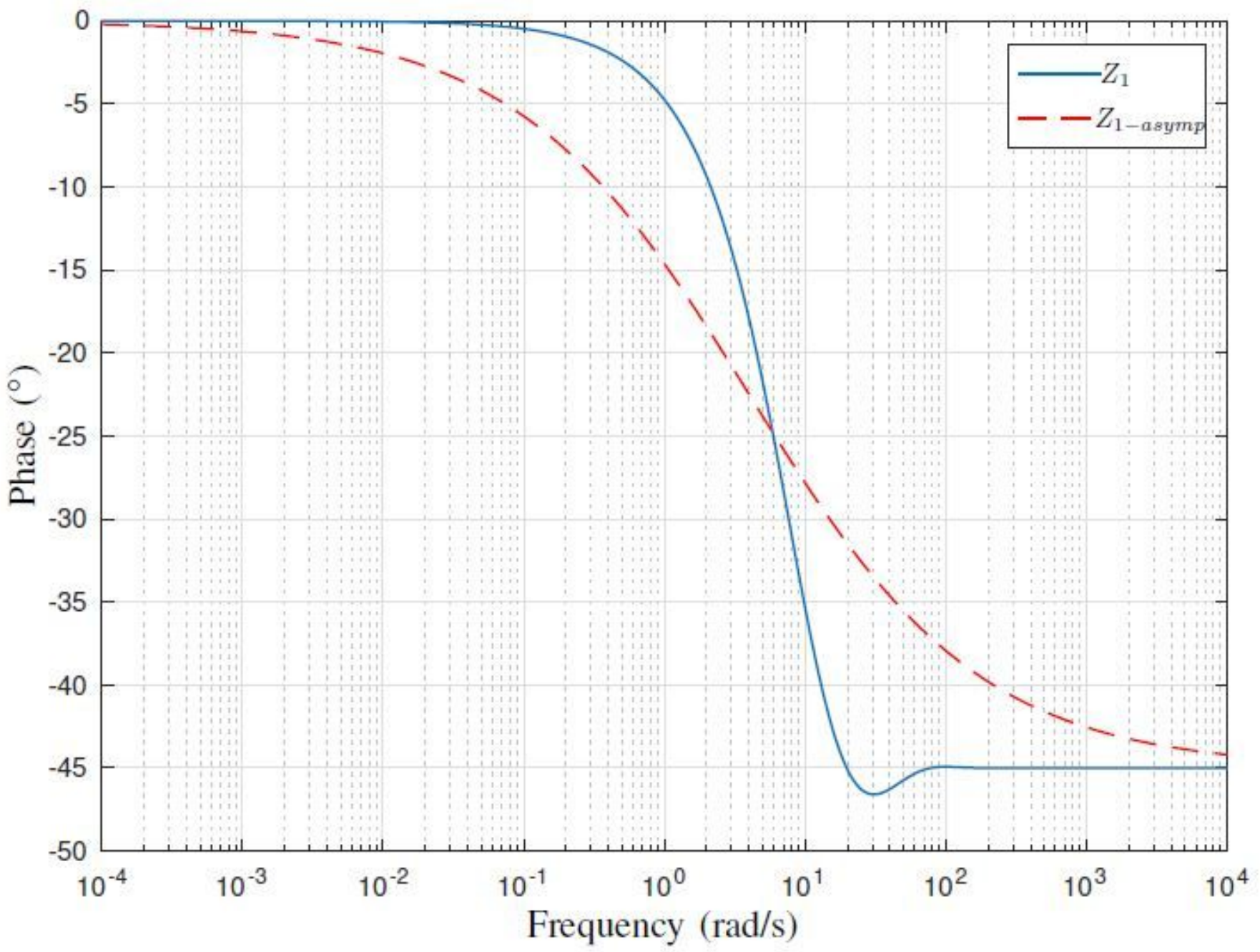

Figure 4

Z1 phase diagram and its asymptotic approximation 


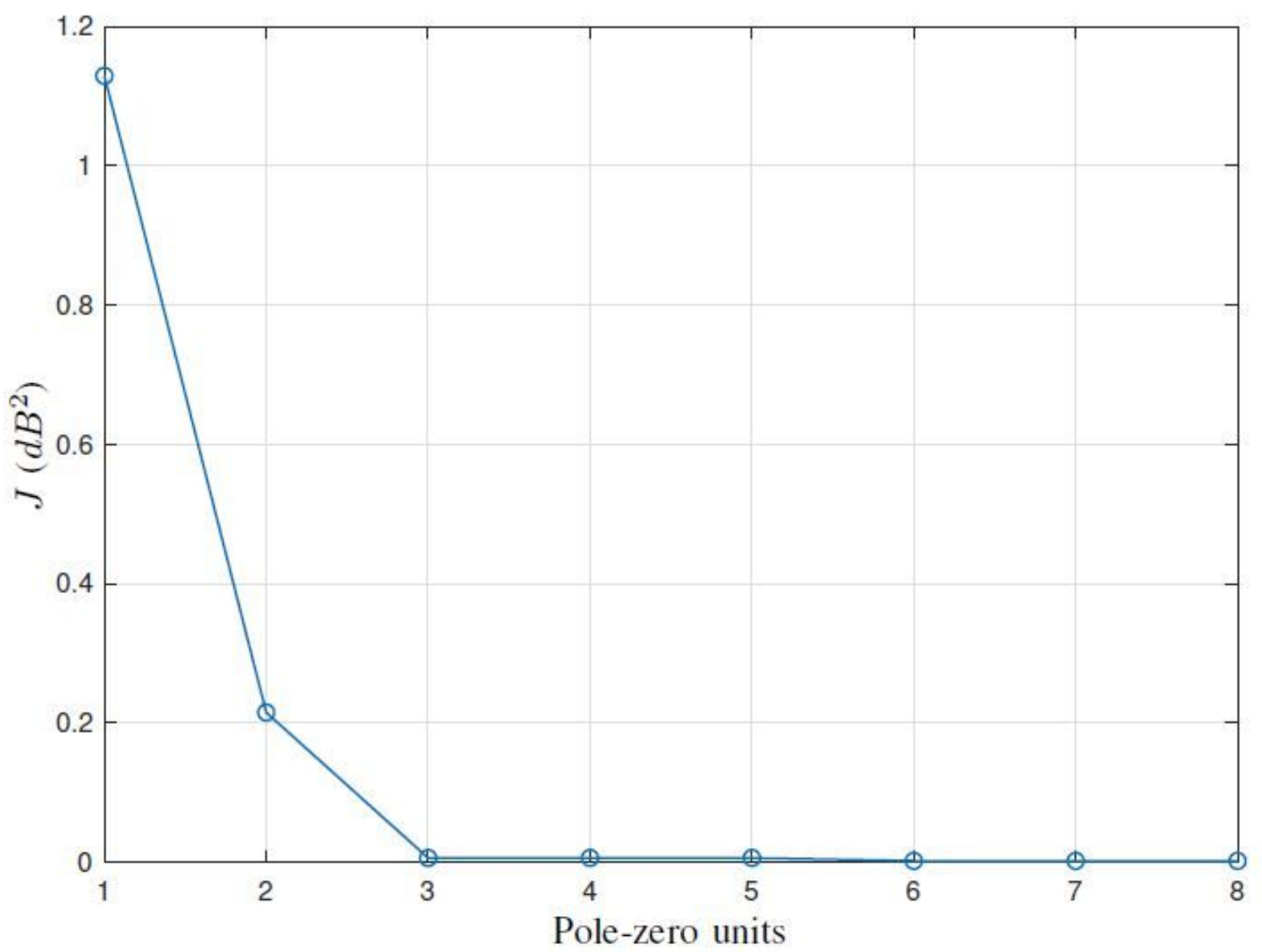

Figure 5

$\mathrm{J}$ criterion with varying Ncells for the series impedance correction 


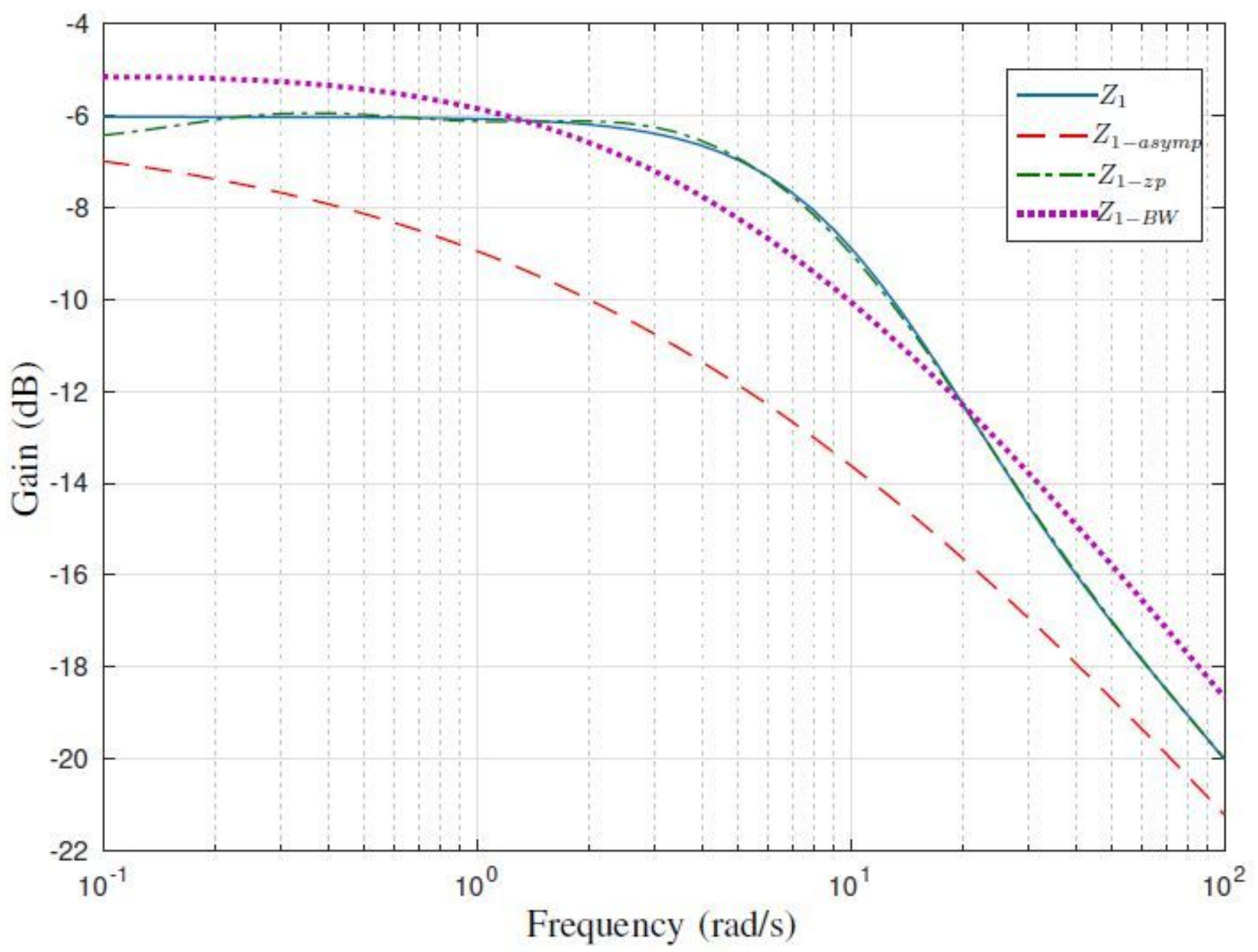

Figure 6

Gain diagrams of $Z 1$ and its approximations 


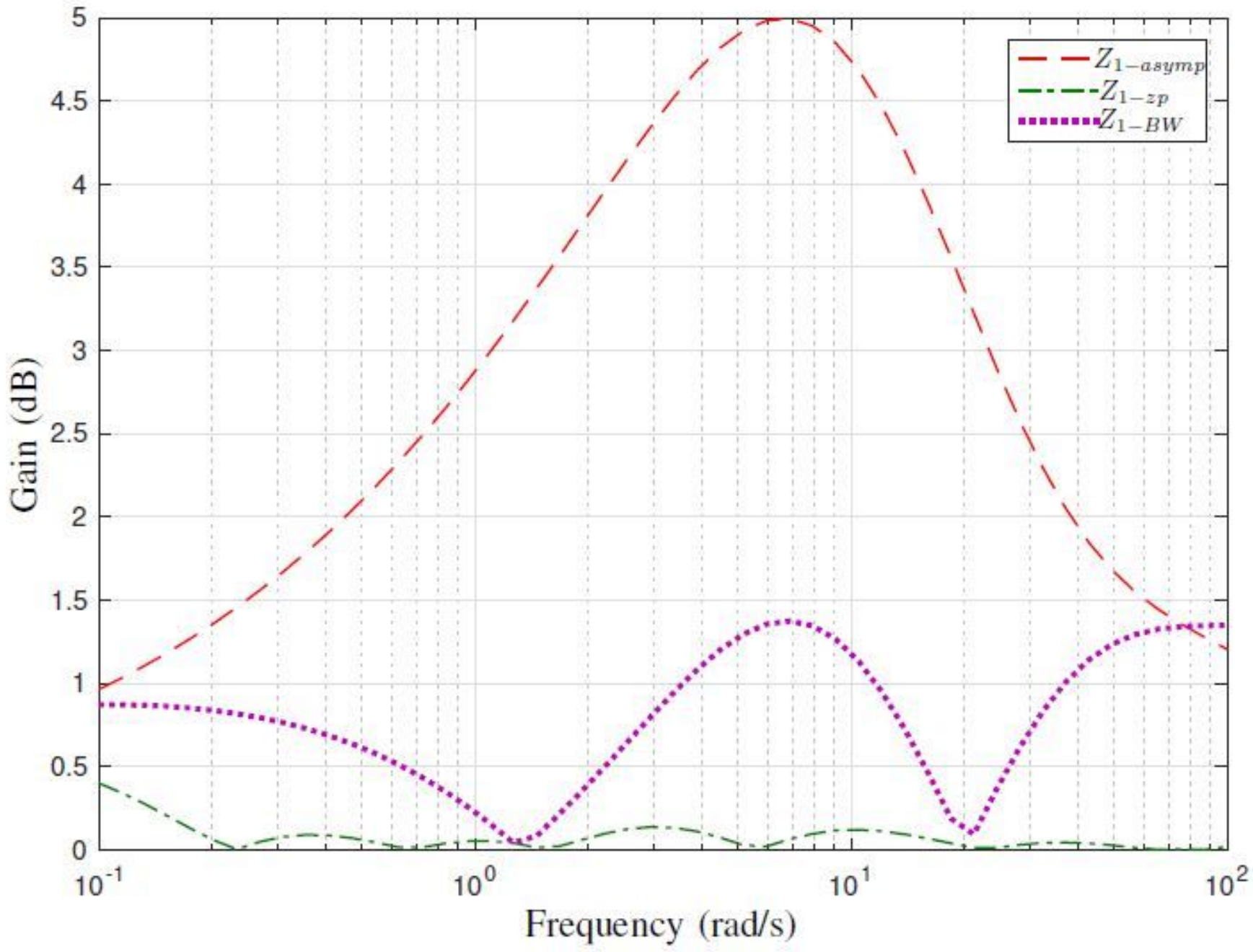

Figure 7

please see the manuscript file for the full caption 


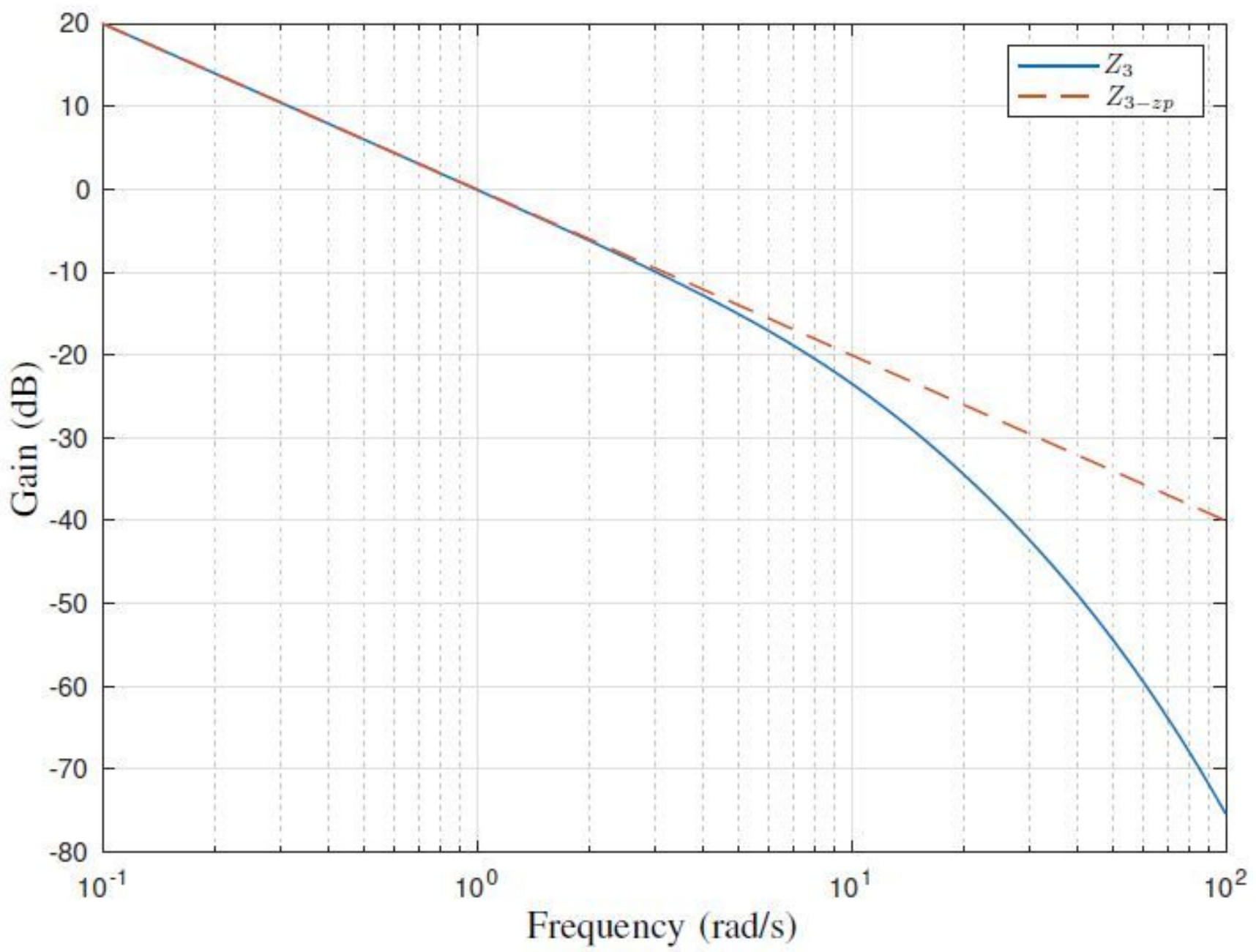

Figure 8

Z3 gain diagram and its capacitance approximation 


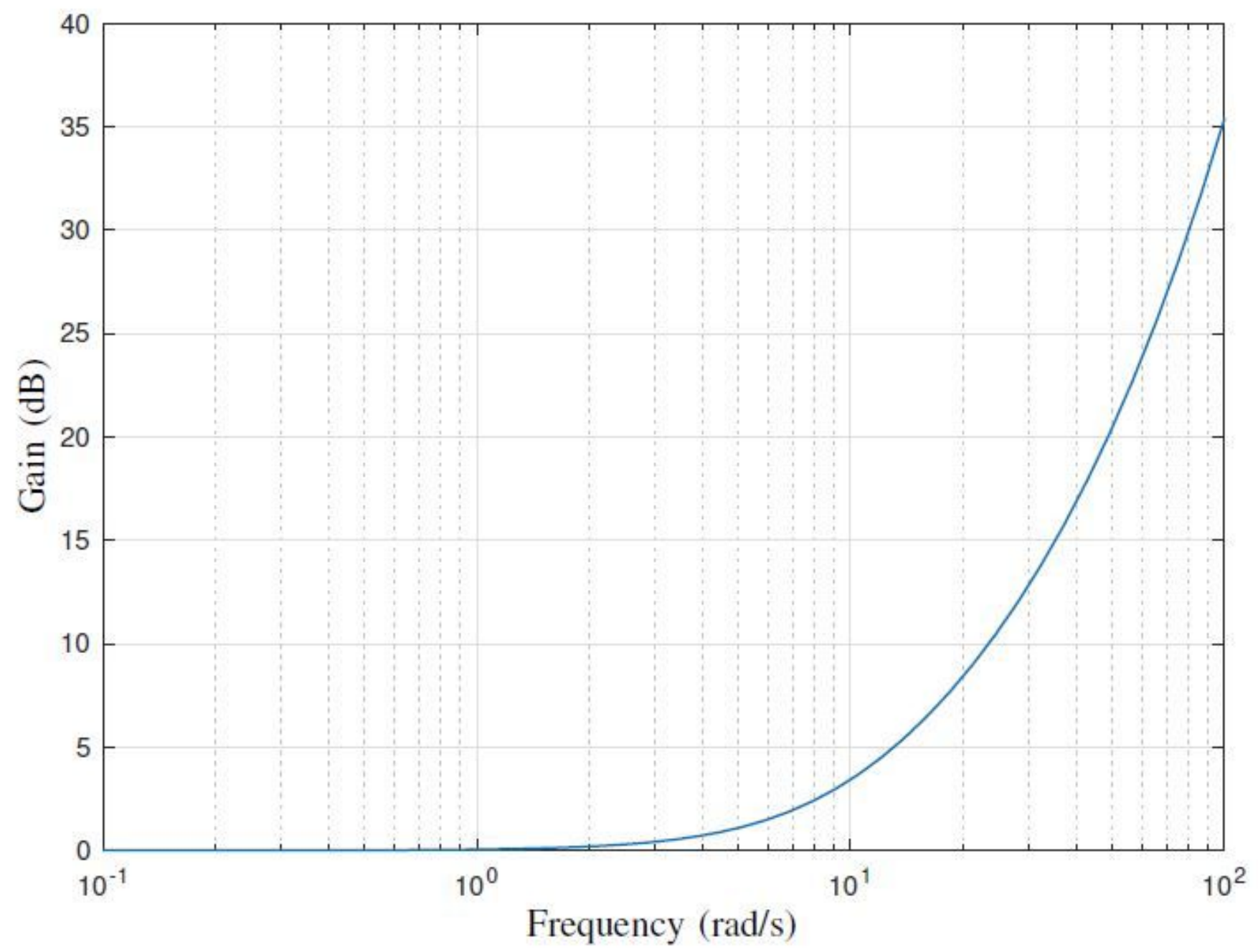

Figure 9

please see the manuscript file for the full caption 


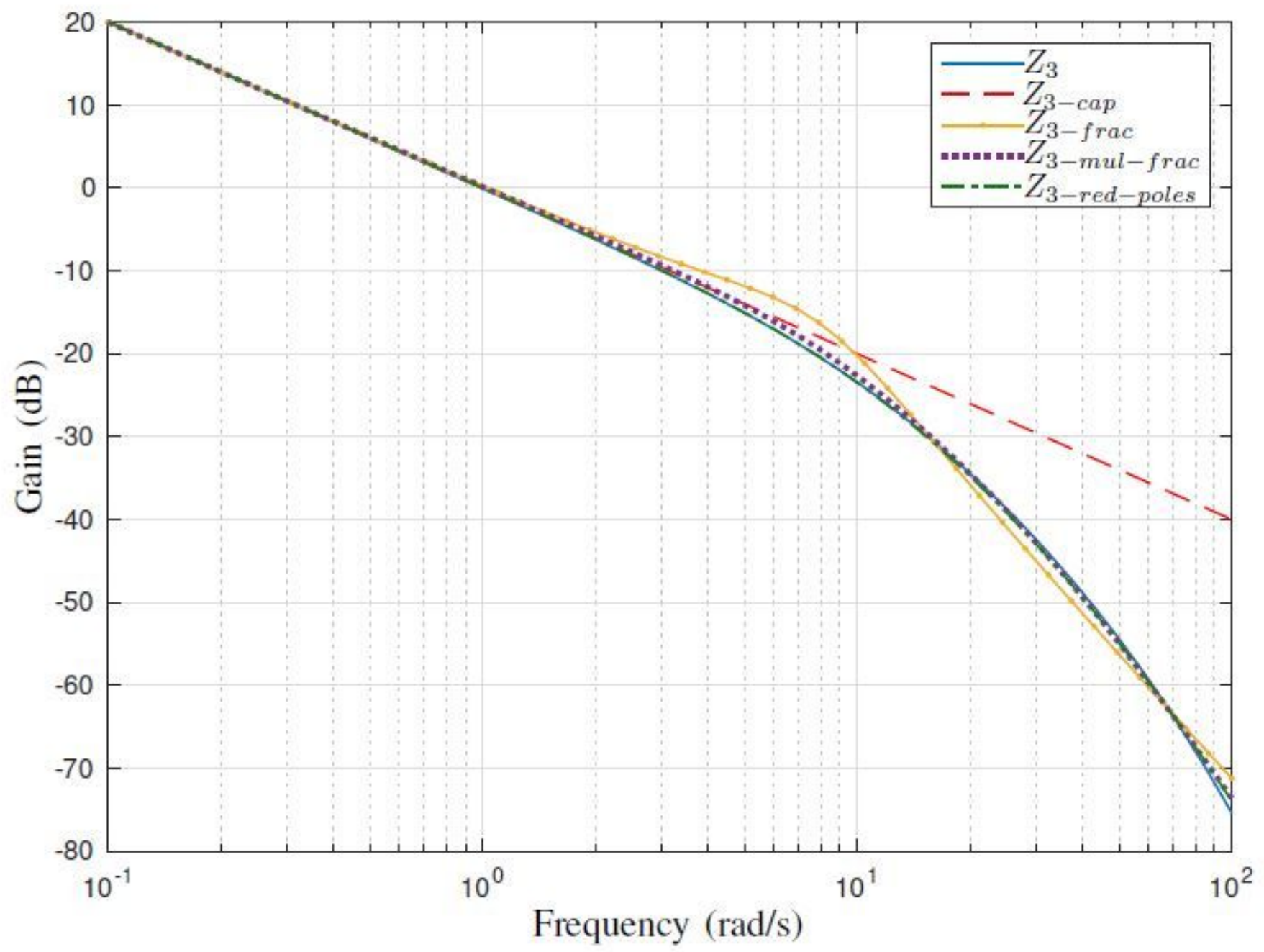

Figure 10

please see the manuscript file for the full caption 


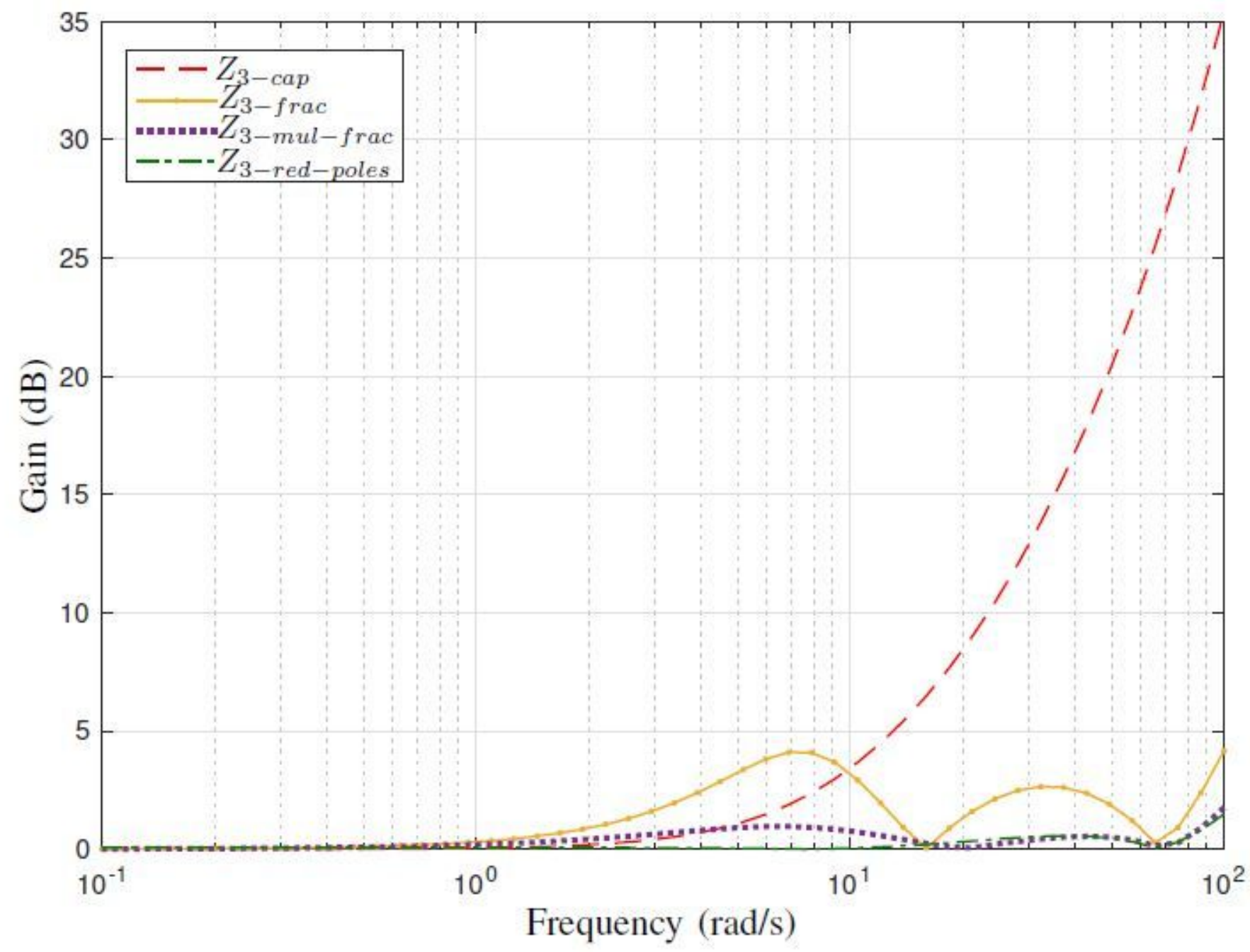

Figure 11

please see the manuscript file for the full caption 


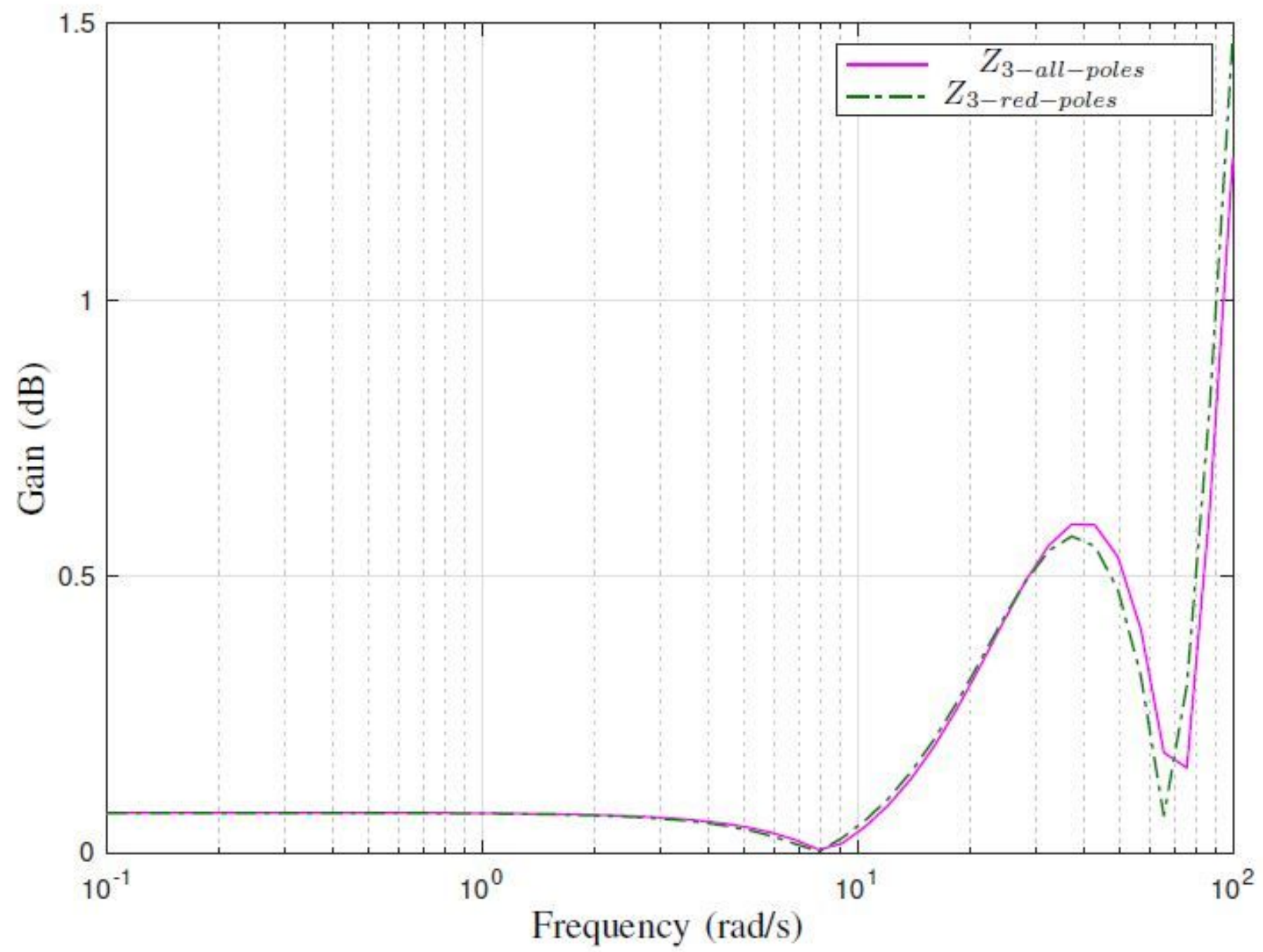

Figure 12

please see the manuscript file for the full caption 


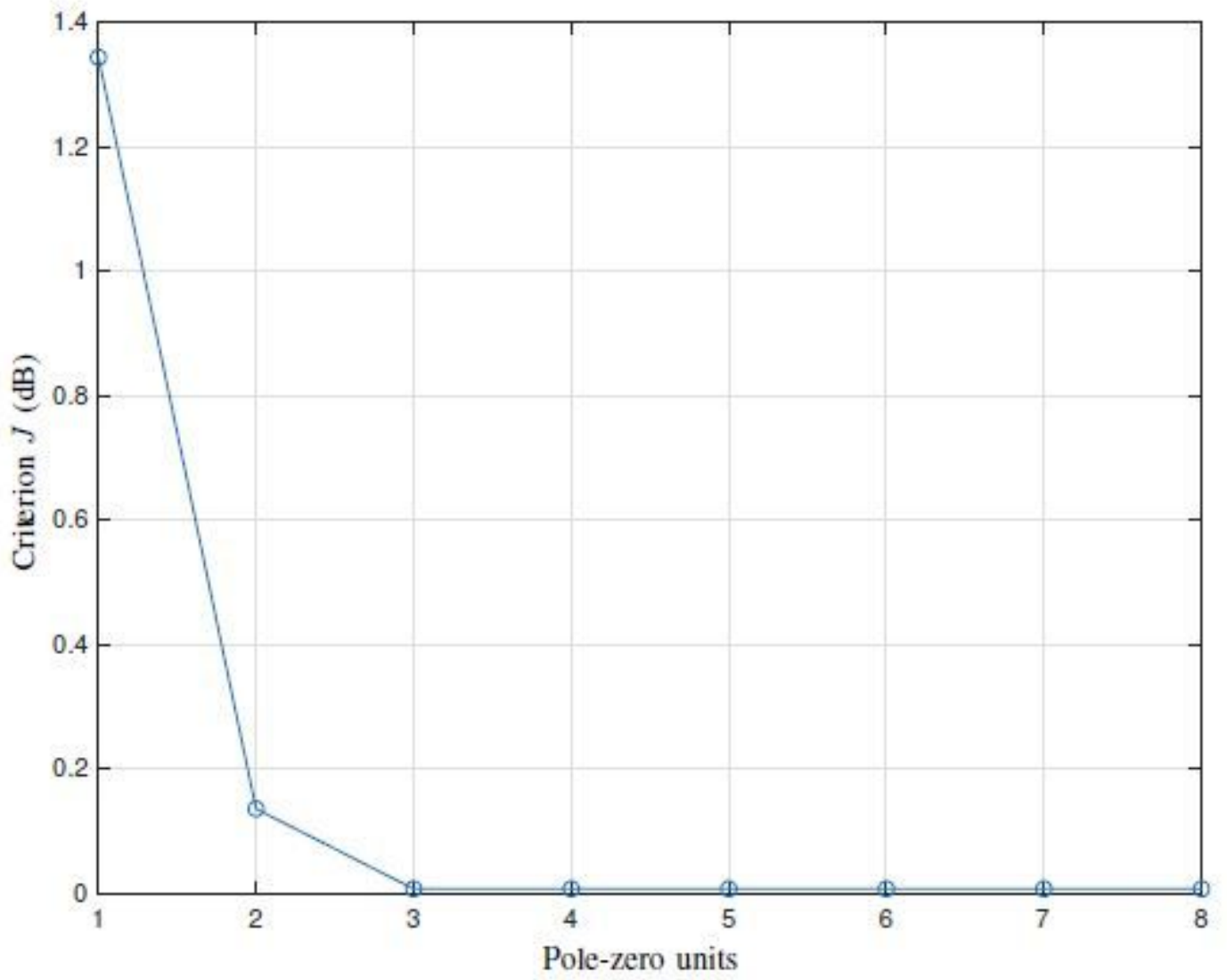

Figure 13

$\mathrm{J}$ criterion versus pole-zero units for a human bronchus

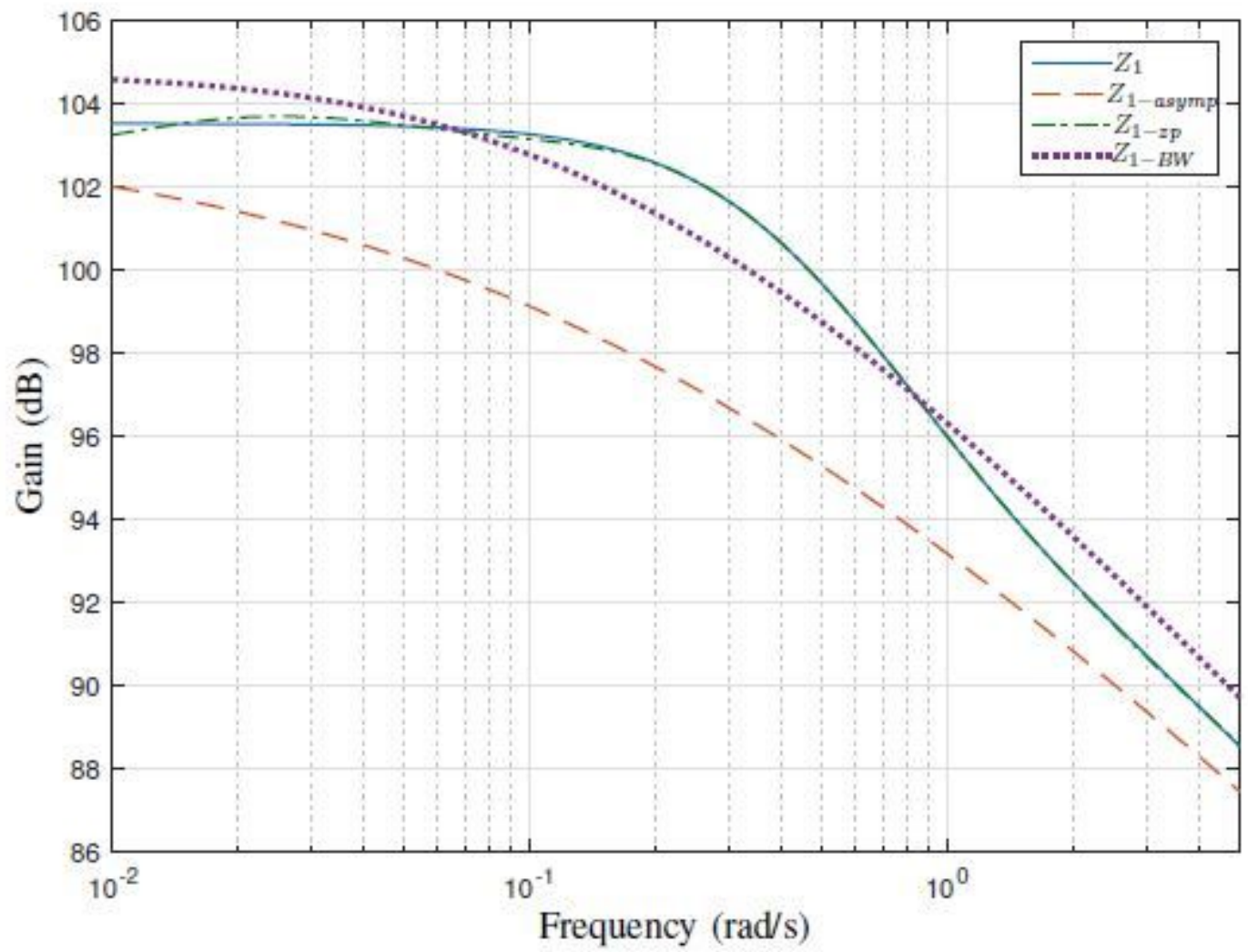


Figure 14

Z1 approximation gains for a human bronchus

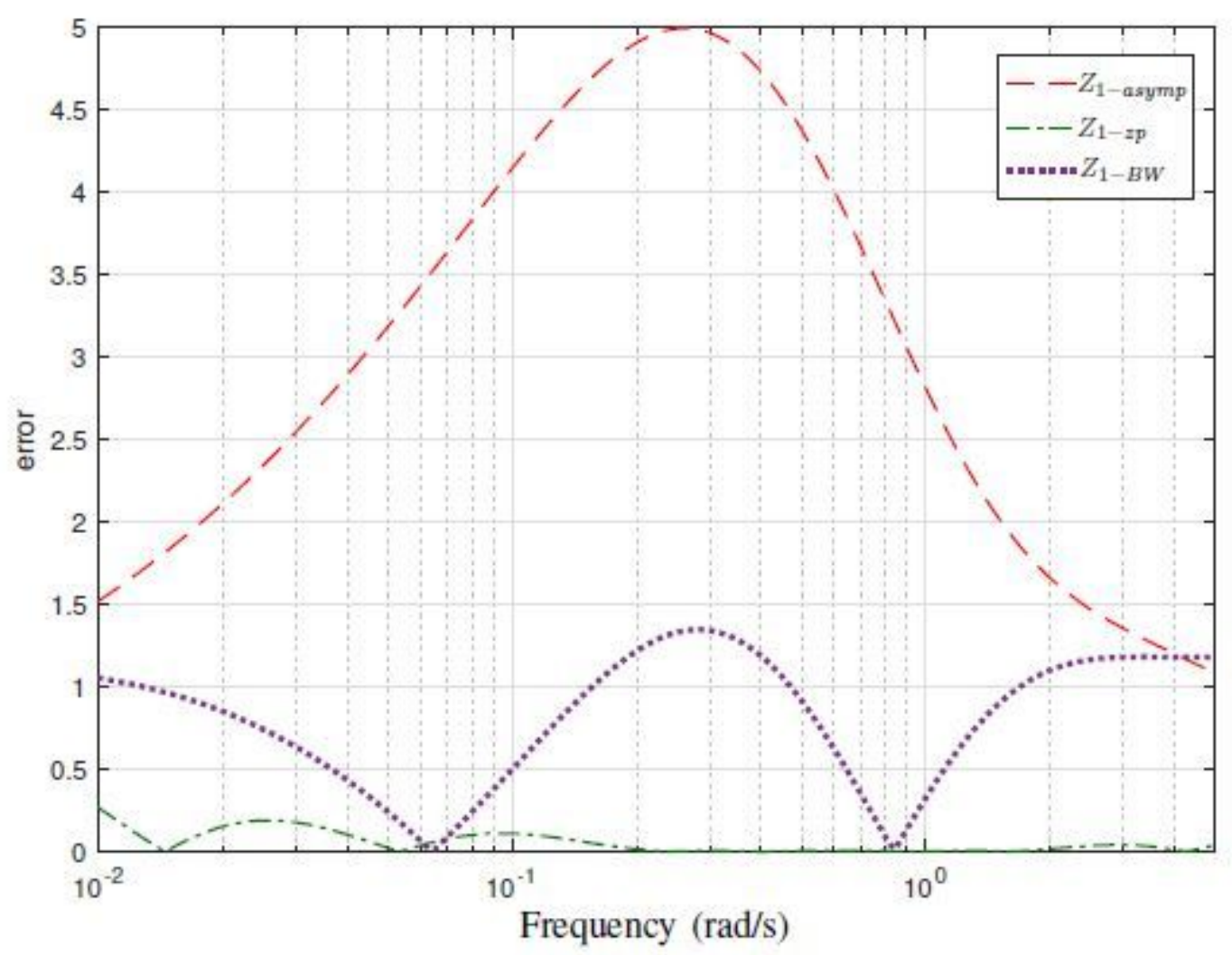

Figure 15

please see the manuscript file for the full caption 


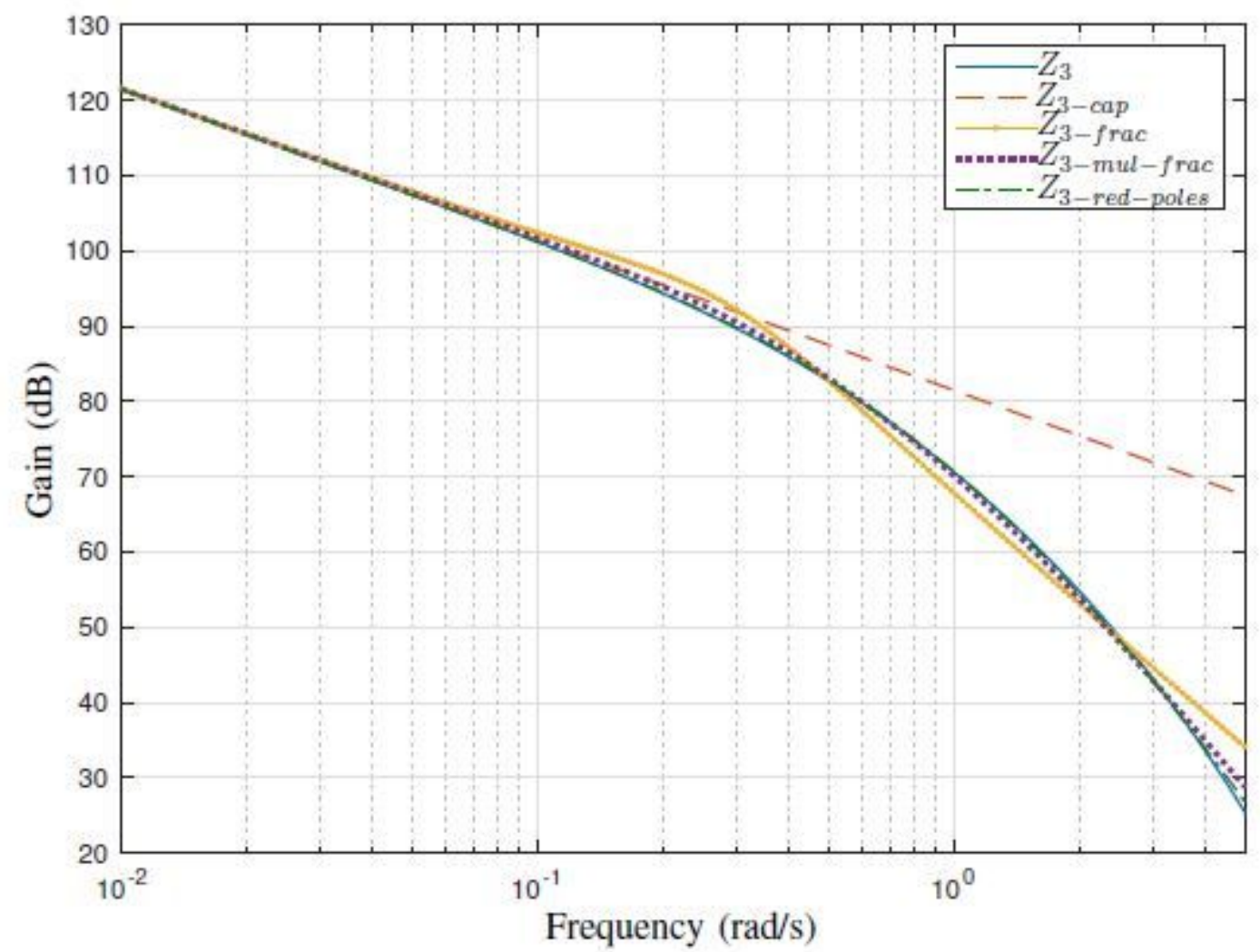

Figure 16

Gain diagrams of $Z 3$ and its approximations for a human bronchus

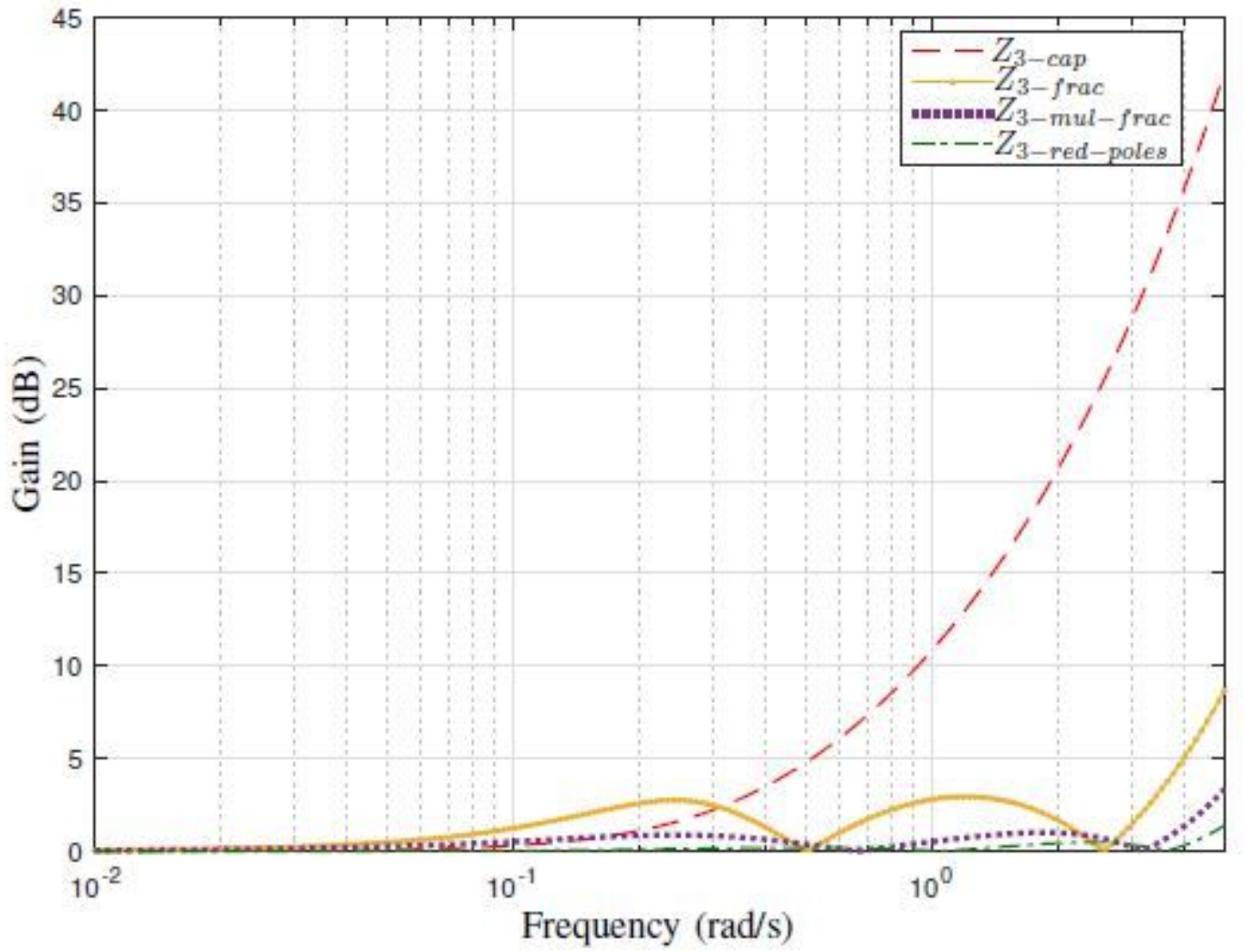


Figure 17

please see the manuscript file for the full caption 